\title{
Karl-Joachim Hölkeskamp
}

\section{Eine politische Kultur (in) der Krise?}

\section{Gemäßigt radikale Vorbemerkungen zum kategorischen Imperativ der Konzepte}

\begin{abstract}
„Die Geschichtsphilosophen haben die Welt nur verschieden verändert; es kömmt darauf an, sie zu verschonen.“
\end{abstract}

(Odo Marquard, Schwierigkeiten mit der Geschichtsphilosophie [Frankfurt 1982] 13)

\section{1. ,Decline` oder ,Fall‘: Traditionen und Positionen}

Es ist natürlich kein Zufall und auch keine beliebige Laune, daß der Untertitel des vorliegenden Bandes auf Erich Gruens gleichnamiges Buch anspielt ${ }^{1}$. Bekanntlich hat er darin - in Auseinandersetzung mit Ronald Symes „Römischer Revolution“ 2 und (eher implizit) mit Christian Meiers These von der lange vor ihrem akuten Ausbruch strukturell angelegten und sich als „autonomer Prozeß“ entfaltenden „Krise ohne Alternative“3 - einen Gegenentwurf zu solchen Modellen entwickelt,

1 The Last Generation of the Roman Republic (Berkeley 1974, ${ }^{2} 1995$, mit einer ausführlichen, allerdings etwas selektiv dokumentierten Einleitung, VII-XXI).

2 Die römische Revolution. Revidierte Neuausgabe, hg. von Christoph Selzer, Uwe Walter (Stuttgart 2003, zuerst Oxford 1939); vgl. dazu Hartmut Galsterer, A Man, a Book, and a Method: Sir Ronald Syme's Roman Revolution after Fifty Years, in: Kurt A. Raaflaub, Mark Toher (Hgg.), Between Republic and Empire. Interpretations of Augustus and His Principate (Berkeley etc. 1990) 1-20; Uwe Walter, Der Historiker in seiner Zeit: Ronald Syme und die Revolution des Augustus, in: Jörg Spielvogel (Hg.), Res publica reperta. Zur Verfassung und Gesellschaft der römischen Republik und des frühen Prinzipats. Festschrift für Jochen Bleicken ... (Stuttgart 2002) 137-152, sowie zu einzelnen Aspekten die einschlägigen Beiträge in: Adalberto Giovannini (Hg.), La révolution romaine après Ronald Syme. Bilans et perspectives (Genf 2000).

3 Christian Meier, Res publica amissa. Eine Studie zu Verfassung und Geschichte der römischen Republik (Wiesbaden 1966, 2. erweiterte Auflage Frankfurt a. M. 1980, mit einer wichtigen Einführung, darin besonders XLIIIff. zum Konzept der „Krise ohne Alternative“) und dazu die wichtige Rezension von Jochen Bleicken, in: ZRG (RA) 85 (1968) 451-461 (= ders., Gesammelte Schriften, Bd.II, hg. von Frank Goldmann, Markus Seblmeyer, Uwe Walter [Stuttgart 1998] 778788). S. zur Krise der Republik als „autonomem Prozeß“ Christian Meier, Fragen und Thesen zu einer Theorie historischer Prozesse, in: ders., Karl-Georg Faber (Hgg.), Historische Prozesse (Theorie der Geschichte. Beiträge zur Historik, Bd. 2, München 1978) 11-66, hier 34ff. und bereits 
die er für Varianten einer Art Orthodoxie hielt: Keineswegs sei eine anpassungsund reformunfähige res publica amissa letztlich zwangsläufig in Kriegen und Bürgerkriegen endgültig untergegangen und ebenso historisch ,notwendig' durch ein System ersetzt worden, daß den Anforderungen imperialer Herrschaft gewachsen war. Kurz und bündig brachte Gruen seine Kritik an dieser angeblichen Orthodoxie mit ihrer irreführenden Fixierung auf „underlying causes“ und „abstract explanations“ und zugleich seinen Gegenentwurf in einer Formel auf den Punkt: „Civil war caused the fall of the Republic - not vice versa. " ${ }^{4}$ Diese revisionistische Umkehrung versuchte Gruen zu konkretisieren, indem er das Bild eines durch Sullas Reformen sogar stabilisierten Systems der aristokratischen Republik zeichnete, das bis in die letzten Monate vor Caesars Übergang über den Rubicon im Januar 49 v. Chr. durchaus funktionsfähig geblieben sei und zumeist tatsächlich funktioniert hätte - mithin eines Systems, das selbst noch zwischen 80 und 50 v. Chr. viel stärker durch die Kontinuitäten der traditionellen senatorischen Herrschaftspraxis auf allen Ebenen als durch Störungen der Ordnung, Konfrontationen und akute Krisen respektive durch (darauf reagierende) Innovationen geprägt gewesen sei.

Diese radikal revisionistische Sicht des Untergangs der Republik ohne vorherige ,Krise ' hat sich zumindest als ,reine Lehre` nicht allgemein durchgesetzt ${ }^{5}$ - das war eigentlich auch nicht zu erwarten ${ }^{6}$. Aber die implizit dahinter stehenden alten und neuen Fragen - also nicht nur die traditionelle Frage nach der Rolle der Hegel-

dens., Der Alltag des Historikers und die historische Theorie, in: Hans Michael Baumgartner, Jörn Rüsen (Hgg.), Seminar: Geschichte und Theorie. Umrisse einer Historik (Frankfurt 1976) 36-58, hier 49ff. Vgl. dazu etwa Rolf Rilinger, Die Interpretation des Niedergangs der römischen Republik durch „Revolution“ und „Krise ohne Alternative“, in: AKG 64 (1982) 279-306, hier $288 \mathrm{ff}$. (= ders., Ordo und dignitas. Beiträge zur römischen Verfassungs- und Sozialgeschichte, hg. von Tassilo Schmitt, Aloys Winterling [Stuttgart 2007] 123-150, hier 132ff.).

${ }^{4}$ Gruen, Last Generation (wie Anm. 1) 504, vgl. 4f.; $498 \mathrm{ff}$.

${ }^{5}$ Vgl. allerdings Karl-Wilhelm Welwei, Caesars Diktatur, der Prinzipat des Augustus und die Fiktion der historischen Notwendigkeit, in: Gymnasium 103 (1996) 477-497 (= ders., Res publica und Imperium. Kleine Schriften zur römischen Geschichte, hg. von Mischa Meier, Meret Strothmann [Stuttgart 2004] 196-216), der die res publica nach Sulla für grundsätzlich funktionsfähig hielt und die Offenheit der Situation zu Beginn des Bürgerkrieges betonte, sowie - noch radikaler und absichtsvoll einseitig - Klaus Martin Girardet, Politische Verantwortung im Ernstfall. Cicero, die Dictatur und der Dictator Caesar, in: Christian Mueller-Goldingen, Kurt Sier (Hgg.), LENAIKA. Festschrift für Carl Werner Müller ... (Stuttgart etc. 1996) 217-251, hier 224; 247ff. (= ders., Rom auf dem Weg von der Republik zum Prinzipat [Bonn 2007] 199-234, hier 206f.; 229ff.), der den „Putschistenführer und Diktator Caesar" und seine "pathologische Gier nach dignitas", seinen „Machtinstinkt“ und skrupellosen „Machtwillen“ als „den wohl am schwersten wiegenden Krisenfaktor“ bezeichnet hat. Vgl. dazu Jürgen Deininger, Zur Kontroverse über die Lebensfähigkeit der Republik in Rom, in: Peter Kneissl, Volker Losemann (Hgg.), Imperium Romanum. Studien zu Geschichte und Rezeption. Festschrift für Karl Christ zum 75. Geburtstag (Stuttgart 1998) 123-136.

6 Vgl. etwa nur die Rezension von David R. Shackleton Bailey, in: AJPh 96 (1975) 436-443; Jochen Bleicken, Gedanken zum Untergang der römischen Republik (Stuttgart 1995) 22ff. (= ders., Gesammelte Schriften II [wie Anm.3] 683-704, hier 700ff.) und Gruen selbst in der Einleitung zur Neuauflage (wie Anm.1) XXI. Vgl. dazu den Überblick über ähnliche Positionen in den Beiträgen von Uwe Walter und Martin Jehne in diesem Band. 
schen „kolossalen Individualitäten“, nach den Spielräumen ihrer Entfaltung und natürlich nach der historischen Bedeutung Caesars ${ }^{7}$ - waren und sind damit ja weder beantwortet, noch haben sie sich etwa gar von selbst erledigt: Das gilt nicht nur für die Frage nach dem Verhältnis von dignitas-Anspruch, ,Legitimität‘ und ,Legalität ${ }^{`}$ in diesem konkreten, letztlich entscheidenden Fall ${ }^{8}$, sondern auch und insbesondere (aber keineswegs allein) für die grundsätzlichen Fragen nach den Potentialen und Kapazitäten des ,Managements', der Bewältigung oder Einhegung von Konflikten und Krisen eines oligarchischen Herrschaftssystems, das sich doch zuvor jahrhundertelang gerade durch seine Flexibilität und Anpassungsfähigkeit ausgezeichnet hatte. Und das gilt auch und zugleich natürlich für die Frage nach Art, Bedeutung und Rolle der Kontingenz in einem ja eigentlich als systemisch vernetzt zu denkenden komplexen „autonomen Prozeß“9.

Eine erneute, intensive Auseinandersetzung um dieses (und andere) Raster der Konzeptualisierung und Deutung lohnt sich nicht nur vor dem Hintergrund der etwas verkürzt sogenannten ,Millar-Debatte' um den, so Millars absichtsvoll antiquierte Diktion, „politischen Charakter“ der Republik ${ }^{10}$ - darauf wird noch zurückzukommen sein. Auch und vor allem lassen sich aus einem solchen neuen Blick auf eine altbekannte radikale Position à la Gruen heute - auf der Grundlage eines breit fundierten und multiperspektivischen Konzepts der ,politischen Kultur $^{\text {'11, }}$, auf das noch zurückzukommen sein wird - neue Fragen, Ansichten und hoffentlich Einsichten zur Republik in diesem besonders gut bezeugten Zeitalter gewinnen: Wie und auf welchen Ebenen der politischen Kultur der res publica lassen sich Symptome einer ,Krise' feststellen? Denkbare zukünftige Antworten auf diese trügerisch einfache Frage können nicht (mehr) nur darin bestehen, einzelne konkrete Konflikte und ,Krisenherde der Republik' zu identifizieren und ihnen je nach Sichtweise und allgemeinen (oft implizit bleibenden) Vorannahmen über den inhärenten Zusammenhang der einzelnen Faktoren - ein größeres oder geringeres Gewicht in einem (wie auch immer gedachten, jedenfalls) komplexen Gesamtbild zuzumessen. Das Spektrum dieser potentiellen oder akuten Krisenherde und die verschiedenen Ebenen, auf denen sie anzusiedeln sind, ist bekanntlich außerordentlich breit und ebenso vielschichtig und vernetzt wie das Phänomen

\footnotetext{
7 Vgl. zu Hegels Urteil über Caesar als „Geschäftsführer des Weltgeistes“ (und zu dem ähnlichen Caesarbild Theodor Mommsens bis zu den "modernen und modernistischen [?!] Annäherungen“) umfassend Karl Christ, Caesar. Annäherungen an einen Diktator (München 1994) und neuerdings Martin Jebne, History's Alternative Caesars: Julius Caesar and Current Historiography, in: Horst Zander (Hg.), Julius Caesar. New Critical Essays (New York etc. 2005) 59-70.

${ }^{8}$ Vgl. die Beiträge von Martin Jehne und Robert Morstein-Marx in diesem Band.

${ }^{9}$ Vgl. dazu wiederum die Beiträge von Uwe Walter und Martin Jehne in diesem Band. Vgl. zum Konzept der Kontingenz (und demjenigen der ,Diskontinuität') allgemein Ute Daniel, Kompendium Kulturgeschichte. Theorien, Praxis, Schlüsselwörter (Frankfurt $\left.{ }^{3} 2002\right) 419 \mathrm{ff}$.

10 Fergus Millar, The Political Character of the Classical Roman Republic, in: JRS 74 (1984) 1-19 (= ders., Rome, The Greek World and the East, vol. I: The Roman Republic and the Augustan Revolution, ed. by Hannab M. Cotton, Guy M. Rogers [Chapel Hill 2002] 109-142).

${ }^{11}$ Vgl. dazu generell Karl-Joachim Hölkeskamp, Rekonstruktionen einer Republik. Die politische Kultur des antiken Rom und die Forschung der letzten Jahrzehnte (München 2004) $55 \mathrm{ff}$.
} 
der ,Krise“ selbst - hier muß und soll daher nur kurz und holzschnittartig an die bekanntesten (und allgemein mehr oder weniger unstrittigen) Probleme erinnert werden, die sich untereinander vielfach bedingten, miteinander kausal und/oder funktional zusammenhingen oder bloß kontingent zusammentrafen, miteinander interagierten und sich dabei gegenseitig verstärkten ${ }^{12}: \mathrm{Zu}$ diesen Faktoren gehören etwa

- die strukturellen und akuten Kollisionen, die aus den nie ausgeglichenen Widersprüchen in der „gewachsenen Verfassung“ zwischen Organen, ihren jeweiligen und jeweils für sich traditionell als durchaus legitim geltenden Kompetenzen, Handlungsspielräumen und Zuständigkeitsansprüchen resultierten - konkret stehen hier die Träger der (regulären oder außerordentlichen) Imperien einerseits und die Volkstribune andererseits im Mittelpunkt, ihre jeweiligen Funktionen und Rollen und vor allem ihre Handlungsspielräume bzw. deren Grenzen und damit ihr Verhältnis zum Senat ${ }^{13}$;

- das Versagen vor den besonderen An- und Herausforderungen, die sich aus der „Extensivierung“ der aristokratisch-stadtstaatlich verfaßten res publica, der schieren Ausdehnung des Imperium Romanum und der schlicht konkurrenzlosen Weltmachtposition einer imperialen Republik ergaben - wiederum konkret

12 Selbst jede Auswahl einschlägiger Forschungsüberblicke ist notwendig selektiv. S. zu den grundsätzlichen Positionen zuletzt Jochen Bleicken, Geschichte der Römischen Republik (München $\left.{ }^{6} 2004\right) 185 \mathrm{ff}$. mit Bibliographie 299ff. und Robert Morstein-Marx, Nathan Rosenstein, The Transformation of the Republic, in: dies. (Hgg.), A Companion to the Roman Republic (Oxford etc. 2006) 625-637, sowie zu den Stadien der akuten ,Krise' C.F. Conrad, From the Gracchi to the First Civil War (133-70), in: ebd. 167-189; W. Jeffrey Tatum, The Final Crisis (69-44), in: ebd. 190-211, sowie ausführlich Andrew Lintott, Political history, 146-95 B.C.; Robin Seager, Sulla; ders., The rise of Pompey; T. Peter Wiseman, The Senate and the populares, 69-60 B.C.; ders., Caesar, Pompey and Rome, 59-50 B.C., in: CAH² IX (1994) 40-103; 165-207; 208-228; 327-367; 368423, jeweils mit weiteren Nachweisen, sowie die Überblicke von Herbert Heftner, Von den Gracchen bis Sulla. Die römische Republik am Scheideweg 133-78 v. Chr. (Regensburg 2006) (zuverlässig, wenn auch konventionell) und von Bernhard Linke, Die römische Republik von den Gracchen bis Sulla (Darmstadt 2005) (modern und originell). Ältere Forschungspositionen sind außerdem bei Klaus Bringmann, Das Problem einer „Römischen Revolution“, in: GWU 31 (1980) 334-377; Jürgen Deininger, Explaining the Change from Republic to Principate in Rome, in: Comparative Civilizations Review 4 (1980) 77-101; 5 (1980) 96-99; Karl Christ, Der Untergang der römischen Republik in moderner Sicht, in: ders., Römische Geschichte und Wissenschaftsgeschichte, Bd.I (Darmstadt 1982) 134-167, und in: ders., Krise und Untergang der römischen Republik (Darmstadt ${ }^{4} 2000$ ) ausführlich dokumentiert. Der eigentlich durchaus erwähnenswerte Überblick von Mary Beard, Michael Crawford, Rome in the Late Republic. Problems and Interpretations (London ${ }^{2} 1999$ ) leidet an einer extremen anglozentrischen Selektivität der Dokumentation.

13 S. dazu Ernst Badian, Tiberius Gracchus and the Beginning of the Roman Revolution, in: ANRW I 1 (1972) 668-731 und danach etwa Walter Eder, Republicans and Sinners. The Decline of the Roman Republic and the End of a Provisional Arrangement, in: Robert W. Wallace, Edward M. Harris (Hgg.), Transitions to Empire. Essays in Greco-Roman History, 360-146B.C., in honor of Ernst Badian (Norman, Okla 1996) hier bes. 440f.; 447ff.; Lukas Thommen, Das Volkstribunat der späten römischen Republik (Stuttgart 1989); Jobn North, The Constitution of the Roman Republic, in: Companion to the Roman Republic (wie Anm.12) 256-277, hier etwa 270ff. S. auch den Beitrag von Wilfried Nippel in diesem Band. 
fokussiert auf eine scheinbar besonders wichtige Antwort auf diese Herausforderungen, nämlich die außerordentlichen Kommandogewalten im weitesten Sinne, ihre im Sinne der aristokratischen Herrschaftspraxis höchst problematische Verortung im System der „gewachsenen Verfassung“, ihre formale Konstruktion und faktische Kontrolle durch die politische Klasse und deren zentrale Institution, den Senat ${ }^{14}$;

- die Spannungen innerhalb dieser Klasse, deren prekäre interne Balance zwischen hierarchisch-asymmetrischer Rangordnung und symmetrischer Gleichheit der Chancen auf Zugang zu ,Macht', Einfluß, Ressourcen und ,symbolischem Kapi-

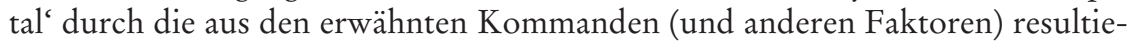
renden Verschiebungen zugunsten der berühmt-berüchtigten ,großen Einzelnen ' nachhaltig gestört wurde ${ }^{15}$ - und dies um so mehr, als ja diese politische Klasse schon auf Grund ihrer Konstitution als einer auf Ämter, Funktionen und Kommanden fixierten Meritokratie gar nicht in der Lage war, diese Spannungen aus sich selbst heraus auszugleichen oder auch nur auszuhalten;

- der (damit vielfach zusammenhängende) schleichende Verlust an Geltung und Bindungswirkung, der die „kollektive Moral“ dieser Klasse, den Haushalt ihrer Werte und Orientierungen und (damit) den darauf beruhenden, akzeptierten ,code of behaviour' mit seinen Konventionen, Verhaltensnormen und -regeln zunehmend erodierte - ein Prozeß, der durch die Versuche der Verfestigung des mos maiorum durch eine formale Verrechtlichung nicht aufgefangen werden konnte ${ }^{16}$;

- die (wiederum aus diesem Prozeß resultierende) quantitative Zunahme und qualitative Eskalation von Übersteigerungen, geradezu mutwillig erscheinenden „Provokationen“ und gezielten „Transgressionen“, die alle Bereiche des ,öffent-

14 Vgl. nur Peter A. Brunt, The Fall of the Roman Republic and Related Essays (Oxford 1988) $68 \mathrm{ff} . ; 81 \mathrm{ff}$. u. o. und die differenzierten Bemerkungen von Jürgen von Ungern-Sternberg, Weltreich und Krise. Äußere Bedingungen für den Niedergang der römischen Republik, in: MH 39 (1982) 254-271 (= ders., Römische Studien. Geschichtsbewußtsein - Zeitalter der Gracchen - Krise der Republik [München etc. 2006] 372-389); Ernst Baltrusch, Auf dem Weg zum Prinzipat: Die Entwicklung der republikanischen Herrschaftspolitik von Sulla bis Pompeius (88-62 v. Chr.), in: Res publica reperta (wie Anm. 2) 245-262; Linke, Die römische Republik (wie Anm. 12) $7 \mathrm{ff}$.; $139 \mathrm{ff}$. Vgl. zu den Konzepten der „Extensivierung“ einerseits und der „gewachsenen Verfassung“ andererseits: Meier, Res publica amissa (wie Anm.3) 64ff. bzw. XXIVff.; 56ff.; 119f. u.ö.

15 Vgl. dazu etwa den Beitrag von Hans Beck in diesem Band.

16 Vgl. dazu etwa Meier, Res publica amissa (wie Anm.3) 57ff.; 297f.; 306 u. ö. S. zum mos maiorum und seiner "Jurifizierung" Jochen Bleicken, Lex publica. Gesetz und Recht in der römischen Republik (Berlin etc. 1975) 387ff., vgl. $371 \mathrm{ff}$. u. ö. (und zu diesem grundlegenden Werk die Rezensionen von Werner Dahlheim, in: GGA 229 [1977] 163-186, und Christian Meier, in: ZRG [RA] 95 [1978] 378-390); Karl-Joachim Hölkeskamp, Exempla und mos maiorum: Überlegungen zum kollektiven Gedächtnis der Nobilität, in: Hans-Joachim Gebrke, Astrid Möller (Hgg.), Vergangenheit und Lebenswelt. Soziale Kommunikation, Traditionsbildung und historisches Bewußtsein (Tübingen 1996) 301-338, hier 316ff.; 327f. (ergänzte Version in: ders., Senatvs Popvlvsqve Romanvs. Die politische Kultur der Republik - Dimensionen und Deutungen [Stuttgart 2004] 169-198, hier 183ff.; 193ff.), sowie zur Problematik des Begriffs jetzt den Beitrag von Wilfried Nippel in diesem Band. 
lichen' und (nicht wirklich), privaten' Lebens der Elite erfaßte, nicht zuletzt die Medien und Formen aristokratischer Selbstdarstellung ${ }^{17}$ - und die eben auch und gerade in den oben erwähnten akuten Kollisionen zwischen Organen, vor allem zwischen den Trägern verschiedener Amtsgewalten und dem Senat und ihren jeweiligen Durchsetzungs- bzw. Kontrollansprüchen die Grenze zur offenen Regelverletzung überschritten und damit ihrerseits die Erosion der Verbindlichkeit der gesamten hergebrachten Ordnung vorantrieben ${ }^{18}$;

- die Spannungen zwischen Klassen und einzelnen gesellschaftlichen Gruppen etwa zwischen Nobilität und Senatsadel generell einerseits und den Rittern bzw. der Gruppe der Publicanen im besonderen andererseits; zwischen den sozialen Eliten insgesamt einerseits und der plebs urbana, der plebs rustica, den Veteranen andererseits und nicht zuletzt zwischen Römern und Italikern ${ }^{19}$;

17 Vgl. zu diesen Kategorien neuerdings Tonio Hölscher, Provokation und Transgression als politischer Habitus in der späten römischen Republik, in: MDAI (R) 111 (2004) 83-104, hier vor allem 84f., und seinen Beitrag in diesem Band, sowie zu einzelnen Aspekten des aristokratischen Lebensstils etwa Elke Stein-Hölkeskamp, Ciceronische Convivia: der rastlose Republikaner und die zügellosen Zecher, in: Hermes 129 (2001) 362-376, hier bes. 370ff.; Henner von Hesberg, Die Häuser der Senatoren in Rom: gesellschaftliche und politische Funktion, in: Werner Eck, Matthäus Heil (Hgg.), Senatores populi Romani. Realität und mediale Präsentation einer Führungsschicht (Stuttgart 2005) 19-52. Vgl. zu den verschiedenen Ebenen der Selbstdarstellung etwa auch Dirk Schlinkert, Den Sieger ehren. Der Diktator Caesar und der Senat nach Pharsalos, in: Theodora Hantos, Gustav A. Lebmann (Hgg.), Althistorisches Kolloquium aus Anlaß des 70. Geburtstags von Jochen Bleicken (Stuttgart 1998) 153-186.

Der auf dem Titel abgebildete Denarius ist dafür ein interessantes frühes Beispiel (Vorderseite: L • MANLI PRO • Q - Darstellung des Kopfes der Roma; Rückseite: L • SVLLA • IM - L. Cornelius Sulla fährt als Imperator in einer Triumphalquadriga und wird von der fliegenden Victoria bekränzt): Im Jahr 82 v. Chr. ließ der Proquaestor L. Manlius Torquatus als Münzmeister Denare und Aurei emittieren, welche die Triumphe Sullas feierten, die diesem wegen seiner Siege über Mithridates und die Samniten am Collinischen Tor zuerkannt worden waren. Die Rückseitenlegende macht deutlich, daß es sich bei dem Dargestellten um Sulla selbst handelt. Damit ist diese Münze eines der frühesten Beispiele für die Darstellung einer noch lebenden Person auf römischen Münzen. Diese Innovation (oder ,Transgression` - vgl. den Beitrag von Tonio Hölscher) - die außergewöhnliche Herausstellung des Imperators Sulla - kontrastierte mit dem konventionellen Vorderseitenbild der Roma, welches an die gemeinsame Sache appellierte und damit gerade die Eingebundenheit Sullas in den Kontext seiner Standesgenossen betonen sollte.

$18 \mathrm{Vgl}$. zur Bedeutung gewalttätiger Störungen des ,regulären' politischen Prozesses für die „Überforderung der republikanischen Ordnung“ insgesamt vor allem Wilfried Nippel, Aufruhr und „Polizei“ in der römischen Republik (Stuttgart 1988) 71ff.; 108ff.; ders., Public Order in Ancient Rome (Cambridge 1995) 47ff.; 70ff.; Paul J.J. Vanderbroeck, Popular Leadership and Collective Behavior in the Late Roman Republic (80-50 B.C.) (Amsterdam 1987); sowie Jean-Michel David, Conformisme et transgression: à propos du tribunat de la plèbe à la fin de la République romaine, in: Klio 75 (1993) 219.

19 Vgl. dazu insgesamt etwa Brunt, Fall of the Roman Republic (wie Anm.14) $68 \mathrm{ff}$.; $93 \mathrm{ff}$.; $144 \mathrm{ff}$. u.ö.; Bleicken, Gedanken zum Untergang der römischen Republik (wie Anm.6) 14ff. (= ders., Gesammelte Schriften II [wie Anm.3] 692ff.); s. zu den Publicanen insbesondere Ernst Badian, Zöllner und Sünder. Unternehmer im Dienst der römischen Republik (Darmstadt 1997, zuerst 1972); zu den Italikern: Emilio Gabba, Rome and Italy: the Social War, in: CAH ${ }^{2}$ IX (1994) 104128 und die revisionistische Perspektive von Henrik Mouritsen, Italian Unification. A Study in Ancient and Modern Historiography (London 1998) bes. $87 \mathrm{ff} . ; 109 \mathrm{ff}$., sowie neuerdings die ein- 
- die massiven sozialen Gravamina, die die genannten Spannungen teils verschärften, teils überhaupt erst generierten und die schon vor dem Hintergrund eines immer steileren Gefälles zwischen Eliten und breiten Schichten des populus Romanus desto schwerer wogen, je länger sie ungeregelt blieben - darunter das ebenso regelmäßig auftretende wie politisch höchst sensible Problem der Versorgung der erwähnten Veteranen ${ }^{20}$;

- der irreversible Verlust an Legitimität der politischen Ordnung insgesamt und des Senats als ihres zentralen Lenkungsorgans im besonderen ${ }^{21}$, der aus einer Mischung aus passiver Handlungsunfähigkeit und aktiver Verhinderung der notwendigen Reformen zur Behebung der genannten drängenden Probleme resultierte - und der damit auch die ,Macht` des Senats zur Kontrolle bzw. Disziplinierung der, großen Einzelnen' rapide verfallen ließ.

\section{Krise der Politik und/oder Politik (in) der Krise: Konzepte und Kategorien}

Die Auflistung der an sich ja längst bekannten Krisenherde ließe sich natürlich noch fortsetzen - eine solche Übung, die ohnehin nie ganz befriedigend sein kann, führt aber hier kaum weiter, zumal eine bloße Akkumulation den dabei zumeist auch angestellten durchaus differenzierten und bedenkenswerten Versuchen ihrer Verortung in komplexen Struktur-, Geschehens- und Entwicklungszusammenhängen nicht gerecht werden kann. Vor allem aber soll hier auf keinen Fall behauptet werden, daß diese (und überhaupt alle bisherigen) Erklärungsansätze überholt oder gar gänzlich irrelevant wären - im Gegenteil: Die in den letzten Jahrzehnten angestellten Versuche, einzelne chronische Konflikte, konkrete Kollisionen und notorische Krisenherde als Ursachen, Bedingungen und/oder antreibende Impulse der ,Krise der Republik namhaft zu machen, müssen die Ausgangsbasis für jeden weiteren (zugegebenermaßen höchst ambitionierten) Versuch bilden, ein gewissermaßen integratives Modell und ein entsprechendes Raster an Kategorien und Konzepten zu einer deskriptiven Erfassung, systematischen Analyse und holistischen ,Erklärung' der Krise und des Untergangs der Republik zu entfalten.

Allerdings reicht es eben auch nicht mehr aus, eine gegenseitige vernetzte Bedingtheit der verschiedenen Faktoren bloß zu konstatieren und in einem einfachen, gewissermaßen eindimensionalen Kräfteparallelogramm darzustellen. Die neuen Fragen nach der systemischen Vernetzung von Strukturen und Kontingen-

schlägigen Beiträge in: Martin Jehne, Rene Pfeilschifter (Hgg.), Herrschaft ohne Integration? Rom und Italien in republikanischer Zeit (Frankfurt 2006).

${ }^{20} \mathrm{Vgl}$. dazu insbesondere Brunt, Fall of the Roman Republic (wie Anm. 14) 73ff.; 240ff.; Henrik Mouritsen, Plebs and Politics in the Late Roman Republic (Cambridge 2001).

$21 \mathrm{Vgl}$. dazu vor allem Jürgen von Undern-Sternberg, Die Legitimitätskrise der römischen Republik, in: HZ 266 (1998) 607-624 (= ders., Römische Studien [wie Anm. 14] 390-404). 
zen, Voraussetzungen und Bedingungen, Ursachen und Anlässen der ,Krise‘ müssen jetzt auf neue Weise und geradezu radikal ,grundsätzlich' ansetzen - im metaphorischen wie pragmatischen Sinne dieses Begriffs. Die erste konkrete Frage in diese Richtung muß mithin etwa so lauten: Welchen ,Grad der Grundsätzlichkeit` hatten die erwähnten strukturellen Gegensätze, offenen Konflikte und (explizit) strittigen Gegenstände überhaupt? Daraus ergibt sich notwendig bereits die weitere Frage: Gab es daneben womöglich drängende Probleme von durchaus ,grundsätzlicher' Bedeutung, die dennoch gar nicht zum Gegenstand offener Kontroversen und (damit) von ,Politik‘ als Entscheidungshandeln wurden oder werden konnten, weil sie im Rahmen des politischen Systems überhaupt nicht thematisierbar respektive ,politisierbar' waren²? Wie steht es überhaupt mit der Wahrnehmung bzw. der Wahrnehmbarkeit der Probleme durch die Zeitgenossen - als Voraussetzung ihrer Politisierbarkeit ${ }^{23}$ ? Welchen ,Grad' - oder auch: welche Qualität - der ,Grundsätzlichkeit‘ müssen diese Gegensätze und Konflikte eigentlich annehmen, damit sinnvollerweise von einer ,Krise‘ die Rede sein kann? Was wollen wir überhaupt unter einer ,Krise` verstehen - geht eine Definition des Konzeptes als dauerhafte Überlastung respektive dabei irreversibel und irreparabel erodierende Kapazität zur Kanalisierung und Bewältigung von Konflikten schon zu weit? Noch einmal anders gefragt: Ist eine ,Krise‘ immer eine plötzliche Herausforderung oder ein mittel- bzw. langfristiger Prozeß, durch die bzw. in dessen Verlauf die Regelungskapazität eines gegebenen gesellschaftlichen Systems notwendig überfordert wird - oder müssen wir nicht regelmäßig auch nach dem ,Grad' der Fähigkeit eines solchen Systems zur Anpassung, zur Invention und Innovation oder gar zum Wechsel der hergebrachten politischen, sozialen und kulturellen Paradigmen fragen?

Und selbst wenn man schließlich doch (wieder) zu dem Schluß kommen sollte, daß sich in der ,letzten Generation der Republik' das Potential an Flexibilität, die spezifischen Kapazitäten zur Anpassung und Erneuerung tatsächlich erschöpft hatten - gerade dann wird die Frage nach den Ursachen und Bedingungen dieser Erschöpfung um so drängender. Denn immerhin hatte die im Senat versammelte

${ }^{22}$ Diese Konzepte habe ich seinerzeit entwickelt, um jenes spezifische Beschreibungsraster und Erklärungspotential, das Christian Meiers Theorie der ,Parteiungsbildung' in vormodernen Gesellschaften enthielt, für die (frühe) Geschichte der römischen Republik fruchtbar zu machen: Ch. M., Alltag des Historikers (wie Anm. 3) 39ff.; ders., Res publica amissa (wie Anm.3) XXXIIff.; LIIIff.; ders., Introduction à l'anthropologie politique de l'Antiquité classique (Paris 1984) 45ff.; $63 \mathrm{ff}$.; Karl-Joachim Hölkeskamp, Die Entstehung der Nobilität. Studien zur sozialen und politischen Geschichte der Römischen Republik im 4. Jh. v. Chr. (Stuttgart 1987) 14ff. Vgl. zum Konzept der ,Krise‘ außerdem Hinnerk Brubns, Crise de la République romaine? Quelle crise?, in: Silvie Franchet d'Espèrey, Valérie Fromentin, Sophie Gotteland, Jean-Michel Roddaz (Hgg.), Fondements et crises du pouvoir (Bordeaux 2003) 365-378; Jean-Michel David, Ce que la crise révèle, in: ebd. 451-455.

${ }^{23}$ Vgl. dazu (und zum Konzept der ,latenten Krise') den Beitrag von Frank Bücher in diesem Band, sowie bereits Martin Jehne, Krisenwahrnehmung und Vorschläge zur Krisenüberwindung bei Cicero, in: Fondements et crises du pouvoir (wie Anm.22) 379-396, mit weiteren Nachweisen. 
politische Klasse der Republik in den gut zwei Jahrhunderten vom 4. bis zum späten 2. Jahrhundert v. Chr. sich nicht zuletzt durch ein hohes Maß an pragmatischpraktischer Lösungsphantasie ausgezeichnet, die sie zum Aufbau und vor allem zur permanenten Weiterentwicklung eines wachsenden Repertoires an Exempeln erfolgreicher Problemlösung, an Regeln zur Einhegung von Konflikten und an konkreten Instrumenten und Strategien der Bewältigung immer neuer Herausforderungen befähigt hatte: Einerseits hatte sich diese eigentümliche Offenheit für praktische, mithin ebenso praktikable wie für die Beteiligten akzeptable Regelungen bereits bei der Überwindung des sogenannten ,Ständekampfes` und der Etablierung der erwähnten politischen Klasse selbst in einer Serie von situationsbedingt-pragmatischen Lösungen und klugen Kompromissen über die Teilung der Prominenzrollen zwischen Patriziat und plebeischer Elite bewährt. Dieser Vorrat an Lösungen wurde zur Basis für das (flexibel weiterentwickelte, aber grundsätzlich immer noch geltende) Regelwerk zur Einhegung der Spannung zwischen kollektivem Konsens und individueller Konkurrenz in dieser hierarchisch geschichteten, auf Rang und Ehre durch hohes Amt und anerkannte Leistung fixierten ,Meritokratie‘. Und auch in der, letzten Generation' der Republik waren zumindest einige führende Köpfe im Senat offenbar durchaus noch dazu fähig, eine ähnliche pragmatische Lösungsphantasie aufzubringen - das höchst ungewöhnliche und (schon deswegen) interessante ,Kompromiß-Angebot' an den Consul Caesar im Jahre 59 v. Chr. ist dafür ein ebenso vielsagendes wie bezeichnendes Exemplum, übrigens durchaus auch im römischen Sinne dieses Begriffs: Nach seinen rabiaten Rechtsbrüchen wurde Caesar anscheinend eine Art ,Indemnität' angeboten, wenn er nur seine gewaltsam gegen den Willen der Senatsmehrheit durchgesetzten acta noch einmal regelkonform einbringen würde - auf diesem Wege sollte eine nachhaltige und womöglich irreversible Beschädigung eben dieser Regeln, auf denen die Lenkungs-, Kontroll- und Ausgleichsfunktionen des Senats und damit die Grundlage seines kollektiven Regimes ja beruhten, nicht nur verhindert werden, sondern diese Regeln sollten daraus sogar in ihrer Geltung bestätigt und gestärkt hervorgehen ${ }^{24}$. Andererseits hatte sich eine ganz ähnliche Fähigkeit zur ,Erfindung' pragmatischer (oft zunächst nur: provisorischer) Lösungen schon bei der Etablierung und Entfaltung des ebenso originellen wie differenzierten und den jeweiligen Verhältnissen angepaßten Repertoires an Instrumenten und Institutionen zur hegemonialen Kontrolle über Italien und dann zur Herrschaft über die weiten

${ }^{24}$ S. zur Entwicklung im 4. und 3. Jh. etwa Hölkeskamp, Nobilität (wie Anm. 22) 170ff.; $241 \mathrm{ff}$. und passim; ders., Conquest, Competition and Consensus: Roman Expansion in Italy and the Rise of the Nobilitas, in: Historia 42 (1993) 12-39 (ergänzte deutsche Version in: ders., SEnATvs Popvlvsqve Romanvs [wie Anm.16] i I-48); Timothy J. Cornell, The Beginnings of Rome. Italy and Rome from the Bronze Age to the Punic Wars (c. 1000-264 BC) (London etc. 1995) 340ff.; 369ff. Vgl. allgemein noch T. Peter Wiseman, Competition and Co-operation, in: ders. (Hg.), Roman Political Life 90 B.C.-A.D. 69 (Exeter 1985) 3-19; John R. Patterson, Political Life in the City of Rome (Bristol 2000) 29ff. S. zu dem erwähnten Exemplum in Caesars erstem Consulat grundlegend Christian Meier, Das Kompromiß-Angebot an Caesar i.J. 59 v. Chr., ein Beispiel senatorischer, Verfassungspolitik‘, in: MH 32 (1975) 197-208. 
Räume des Imperium Romanum beeindruckend manifestiert - das gilt zumindest grundsätzlich selbst dann, wenn die ,vorletzte Generation‘ der Republik hinsichtlich des Italiker-Problems versagte und wenn das im besten Falle vorsichtige, oft genug aber dilettantisch-gleichgültige bis tyrannisch-korrupte Regime, das die Repräsentanten einer politischen Klasse, die auf Eroberung und Herrschaft fixiert war und wenig für die Routinen des Regierens und den Alltag des Administrierens übrig hatte, in den Provinzen ausübten, ein ungelöstes und aus der Mitte dieser Klasse heraus kaum lösbares Problem war und blieb ${ }^{25}$.

Dennoch bleibt festzuhalten: Vor dem Hintergrund einer aus Flexibilität, Anpassungsfähigkeit und wohl auch -bereitschaft resultierenden, durchaus robusten Stabilität, die über viele Generationen die immer neuen politischen, sozialen und vor allem herrschaftssoziologischen An- und Herausforderungen (zugegebenermaßen nicht immer, aber immer öfter mehr schlecht als recht) auffangen und bewältigen konnte, wird die ,Krise dieses Systems und ihre anscheinend so fatale eigengesetzliche Dynamik auf neue Art erklärungsbedürftig. Vielleicht darf man ein bekanntes Dictum Christian Meiers (heute wieder) einmal ,vom Kopf auf die Füße stellen' und fragen, ob nicht doch die Krise und der Untergang der aristokratischen Republik, insbesondere ihre „bemerkenswerte Wehrlosigkeit“ gegenüber neuen Herausforderungen, das erstaunliche und erklärungsbedürftige Phänomen seien (und eben nicht die erwähnte, natürlich ihrerseits erstaunliche jahrhundertelange relative Stabilität der aristokratischen res publica $)^{26}$. Daraus ergeben sich weitere Fragen, die neu und unvoreingenommen reflektiert oder überhaupt erst einmal genauer formuliert werden müssen: Waren es wirklich die so lange bewährten Regeln und Instrumente der aristokratischen Regierungspraxis, die sich relativ

25 Tatsächlich werden heute eher die „Schwierigkeiten, Widerstände und Widersprüche“ im ,römischen' Italien betont: s. dazu Martin Jehne, Rene Pfeilschifter, Einleitung: zum Charakter der römischen Herrschaft in Italien, in: Herrschaft ohne Integration? (wie Anm.19) 7-22, hier 14. Außerdem sind Kategorien wie ,Integration' und insbesondere ,Romanisierung' (und ihre Implikationen) ins Gerede gekommen (ebd. 12f.; Mouritsen, Unification [wie Anm. 19] 39ff.; $59 \mathrm{ff}$. , jeweils mit weiteren Nachweisen). Auch muß das Regime in den Provinzen aus vielen Gründen eher kritisch gesehen werden. S. zur Entwicklung der Hegemonie in Italien die übrigen Beiträge des erwähnten Sammelbandes, sowie etwa Cornell, The Beginnings of Rome (wie Anm. 24) $345 \mathrm{ff}$;; Jean-Michel David, The Roman Conquest of Italy (Oxford 1996, zuerst franz. 1994) und bereits Hartmut Galsterer, Herrschaft und Verwaltung im republikanischen Italien. Die Beziehungen Roms zu den italischen Gemeinden vom Latinerfrieden 338 v. Chr. bis zum Bundesgenossenkrieg 91 v. Chr. (München 1976); Theodora Hantos, Das römische Bundesgenossensystem in Italien (München 1983); dies., Rom und Italien, in: Althistorisches Kolloquium (wie Anm. 17) 103-120. Vgl. zum Regime in den Provinzen nach wie vor Werner Dablheim, Gewalt und Herrschaft. Das provinziale Herrschaftssystem der römischen Republik (Berlin 1977); Robert Morstein (Kallet)Marx, Hegemony to Empire. The Development of the Roman Imperium in the East from 148 to 62 B.C. (Berkeley etc. 1995) und Raimund Schulz, Herrschaft und Regierung, Roms Regiment in den Provinzen in der Zeit der Republik (Paderborn etc. 1997); Karl-Joachim Hölkeskamp, Herrschaft, Verwaltung und Verwandtes. Prolegomena zu Konzepten und Kategorien, in: Rudolf Haensch, Johannes Heinrichs (Hgg.), Herrschen und Verwalten. Der Alltag der römischen Administration in der Hohen Kaiserzeit (Köln etc. 2007) 1-18, jeweils mit weiteren Nachweisen.

26 Meier, Res publica amissa (wie Anm.3) XV; XXI; XXVI, vgl. dagegen allerdings etwa 301. 
plötzlich als inadäquat oder gar kontraproduktiv erwiesen - und wenn ja, warum eigentlich? Wenn also das Regime der senatorischen Führung überlastet und dann überfordert gewesen sein sollte: War dann die traditionelle stadtstaatlich-oligarchische politische Kultur des republikanischen Rom Ursache oder Bedingung, treibender Impuls oder ,Opfer ‘ einer solchen ,Überforderungskrise` - oder vielleicht alles zugleich? Hat sich in der späten Republik gar eine eigene politisch-gesellschaftliche ,Kultur der Krise‘ entwickelt, die womöglich eines eigenen Modells der Diagnose und Beschreibung bedarf?

Um diesen Fragen auf den Grund zu gehen, kommt es zunächst darauf an, Bilanz zu ziehen, und das heißt aus meiner Sicht:

- die in den letzten drei Jahrzehnten entwickelten theoretischen und methodischen Ansätze und Perspektiven und die bereits erreichten Ergebnisse zu bilanzieren und zu bewerten;

- diese Ansätze bzw. Perspektiven auf ihre eventuellen weiteren Erklärungspotentiale hin zu befragen.

\section{3. „Politische Grammatik“, die neue historische Politikforschung und andere,Wenden': Theorien, Methoden und Kontexte}

Eine solche Bilanz erscheint mir heute wichtig und notwendig, und sie kommt auch zur rechten Zeit - aus verschiedenen, durchaus unterschiedlichen Gründen, auf die ich nun in einer ebenso allgemeinen wie subjektiven und (schon daher) notwendig selektiven ,Eröffnungsbilanz' zur Forschung der letzten Jahrzehnte eingehen möchte ${ }^{27}$. Mittlerweile ist die ,zünftige' Althistorie aus dem Ghetto ihrer traditionellen fachlichen Fixierungen ausgebrochen: Sie hat das enge, tendenziell ahistorische Verständnis von ,Recht' und ,Verfassung' ebenso hinter sich gelassen wie die antiquarische Ausrichtung auf die sattsam bekannten „traités et batailles“ oder ,Haupt- und Staatsaktionen` der ,großen Männer'von Camillus bis zu dem spätrepublikanischen, Dreigestirn' Sulla-Pompeius-Caesar. Unter dem Eindruck der Diskussionen in den historischen Nachbardisziplinen - nicht nur über Gesellschafts-, Struktur- und später Kulturgeschichte, sondern auch über ganz neue Themen, Theorien und Methoden, Darstellungsformen und Erkenntnisziele - hat sich nun auch die Althistorie mehr und mehr um eine Erneuerung des Faches selbst bemüht, also einerseits um eine neue Formulierung spezifisch historischer Fragen und Probleme, und andererseits um eine interdisziplinäre Erweiterung und Neupositionierung im Rahmen - oder genauer: am Schnittpunkt des Koordinatenkreuzes - der historischen und altertumswissenschaftlichen Nachbardisziplinen ${ }^{28}$.

${ }^{27}$ In diesem Zusammenhang besonders wichtig ist die systematische nach ,großen Themen ' angelegte tour d'horizon von Martin Jehne, Methods, Models, and Historiography, in: Companion to the Roman Republic (wie Anm.12) 3-28.

${ }^{28}$ Vgl. dazu Hans-Joachim Gehrke, Zwischen Altertumswissenschaft und Geschichte. Zur Standortbestimmung der Alten Geschichte am Ende des 20.Jahrhunderts, in: Ernst-Richard Schwinge 
Nachdem die Zunft den drängenden, interferierenden, sich partiell verstärkenden und partiell neutralisierenden Wellen oder ,turns' von Postmoderne, Dekonstruktion und ,linguistic turn' einen zähen, hinhaltenden und (in unterschiedlichen Graden) erfolgreichen Widerstand geleistet hat ${ }^{29}$, haben der ,cultural turn' und seine gleichzeitigen oder folgenden kulturwissenschaftlichen ,Teil-Wenden ${ }^{\prime 30}$ zumindest einen Teil des Faches bzw. seiner dafür gewissermaßen anfälligen Vertreter kalt erwischt - und das ist, diese Prognose darf man vielleicht wagen, nicht bloß eine Mode, sondern eine Chance.

Notabene setzt das allerdings ein besonderes Verständnis dieses ,turn“ voraus hier geht es nicht um eine „Neuentdeckung“ oder gar „Neuerfindung“ der ganzen römisch-republikanischen Welt und einen damit immer gleich verbundenen ebenso umfassenden wie herrischen „Einzigartigkeits- und Ausschließlichkeitsanspruch“, den einige notorische Propheten der erwähnten jeweiligen ,turns' erhoben haben, der aber allein schon durch die „inflationär gewordene Rede vom turn“ mittlerweile (glücklicherweise) „unterminiert und ironisiert" worden ist ${ }^{31}$. Hier soll es vielmehr um eine „Raffinierung“ und „Erweiterung der geschichtlichen Wahrnehmungsweisen“ durch „Verschiebungen von Blickwinkeln und Zugängen“ gehen, so daß „bisher nicht oder nur wenig beleuchtete Seiten“ einer komplexen

(Hg.), Die Wissenschaften vom Altertum am Ende des 2.Jahrtausends n. Chr. (Stuttgart etc. 1995) 160-196; Justus Cobet, Alte Geschichte, in: Michael Maurer (Hg.), Aufriß der Historischen Wissenschaften, Bd.1: Epochen (Stuttgart 2005) 14-105; Hölkeskamp, Rekonstruktionen (wie Anm.11) $107 \mathrm{ff}$. und passim.

${ }^{29}$ Vgl. die (durchaus unterschiedlich konzipierten) Überblicke von Ute Daniel, Clio unter Kulturschock. Zu den aktuellen Debatten der Geschichtswissenschaft, in: GWU 48 (1997) 195-219; 259-278; Elizabeth A. Clark, History, Theory, Text. Historians and the Linguistic Turn (Cambridge, Mass. 2004) und Jörg Baberowski, Der Sinn der Geschichte. Geschichtstheorien von Hegel bis Foucault (München 2005) 24ff; 190ff.; 204ff., sowie neuerdings Jürgen Trabant, Zur Einführung: Vom linguistic turn der Geschichte zum historical turn der Linguistik, in: ders., Elisabeth Müller-Luckner (Hgg.) Sprache der Geschichte (München 2005) VII-XXII, sowie die übrigen einschlägigen Beiträge in diesem Band; Richard J. Evans, Fakten und Fiktionen. Über die Grundlagen historischer Erkenntnis (Frankfurt etc. 1999; der programmatische Titel des engl. Originals lautete: In Defence of History [London 1997]); Ernst Hanisch, Die linguistische Wende. Geschichtswissenschaft und Literatur, in: Wolfgang Hardtwig, Hans-Ulrich Webler (Hgg.), Kulturgeschichte Heute (Göttingen 1996) 212-230; Chris Lorenz, Postmoderne Herausforderungen an die Gesellschaftsgeschichte?, in: GuG 24 (1998) 617-632; Hans-Jürgen Goertz, Unsichere Geschichte. Zur Theorie historischer Referentialität (Stuttgart 2001), jeweils mit weiteren Nachweisen, und zuletzt Andreas Kablitz, Geschichte - Tradition - Erinnerung? Wider die Subjektivierung der Geschichte, in: GuG 32 (2006) 220-237. Einen wichtigen Beitrag zur Versachlichung der gelegentlich „außerordentlich schrill“ geführten Debatte um die Geschichtswissenschaft in der ,Postmoderne‘ bietet Daniel, Kompendium Kulturgeschichte (wie Anm.9) $150 \mathrm{ff}$.

30 Vgl. dazu den Überblick von Friedrich Jaeger, Historische Kulturwissenschaft, in: ders., Jürgen Straub (Hgg.), Handbuch der Kulturwissenschaften, Bd.2: Paradigmen und Disziplinen (Stuttgart etc. 2004) 518-545, mit umfangreichen Literaturangaben, die ausführlichen Analysen von Doris Bachmann-Medick, Cultural Turns. Neuorientierungen in den Kulturwissenschaften (Reinbek bei Hamburg 2006), sowie den Überblick von Silvia Serena Tschopp, Wolfgang E.J. Weber, Grundfragen der Kulturgeschichte (Darmstadt 2007) 84ff.

${ }^{11}$ Karl Schlögel, Im Raume lesen wir die Zeit. Über Zivilisationsgeschichte und Geopolitik (München 2003) 68. 
historischen Realität sichtbar werden - im besten Falle kann sich daraus dann eine „Verfeinerung des Registers der Geschichtsschreibung“ ergeben ${ }^{32}$, und zwar, wie gerade in diesem Zusammenhang ausdrücklich betont zu werden verdient, auf der Basis des bereits erreichten, theoretisch reflektierten, methodisch kontrollierten und empirisch fundierten Erkenntnisstandes.

Genau damit sind wir wieder bei der Notwendigkeit, hier und jetzt Bilanz zu ziehen. Schon die Debatte über Charakter und Begriff des römischen ,Imperialismus' war ja eine Art des frühen, Wetterleuchtens', nämlich ein Indiz für das erste Stadium der Öffnung des Faches ${ }^{33}$ - vielleicht sogar mehr und jedenfalls nachhaltiger als die Auseinandersetzung um den Untergang der Republik und die Etablierung des Prinzipats als „Revolution“34. Die Diskussion um die Angemessenheit moderner Begriffe zog notwendig und daher relativ rasch neue, grundsätzliche Fragen nach den Voraussetzungen, Bedingungen und Impulsen der römischen Expansion insgesamt nach sich: Schließlich ging - und geht es immer noch - um die Frage nach der dynamischen Verflechtung zwischen Herrschaft und Integration, imperialer Hegemonie und stadtstaatlicher Struktur, ,Extensivierung', Überdehnung und Desintegration, die wegen ihrer Komplexität neue Probleme der Konzeptualisierung und Darstellung bzw. Darstellbarkeit aufwirft ${ }^{35}$. Gerade hier zeigt

32 Begriffe und Zitate nach Schlögel, Im Raume lesen wir die Zeit (wie Anm.31) 502, vgl. 60ff. u. ö.

33 S. etwa Robert Werner, Das Problem des Imperialismus und die römische Ostpolitik im zweiten Jahrhundert v. Chr., in: ANRW I, 1 (1972) 501-563; Franz Hampl, Das Problem des Aufstiegs Roms zur Weltmacht. Neue Bilanz unter methodisch-kritischen Aspekten, in: ders., Geschichte als kritische Wissenschaft, Bd.3, hg. von Ingomar Weiler (Darmstadt 1977) 48-119; vgl. auch Paul Veyne, Y a-t-il un impérialisme romain?, in: MEFRA 87 (1975) 793-855.

34 Alfred Heuß, Der Untergang der römischen Republik und das Problem der Revolution, in: HZ 182 (1956) 1-28; ders., Das Revolutionsproblem im Spiegel der antiken Geschichte, in: HZ 216 (1973) 1-72, hier 47ff.; 71f. (= ders., Gesammelte Schriften [Stuttgart 1995] Bd. II, 1164-1191 bzw. Bd. I, 500-571, hier 546ff.; 570f.; Karl Ernst Petzold, Römische Revolution oder Krise der römischen Republik?, in: RSA 2 (1972) 229-243; Joachim Molthagen, Rückwirkungen der römischen Expansion. Der Übergang von der Republik zum Prinzipat - eine Revolution?, in: Imanuel Geiss, Rainer Tamchina (Hgg.), Ansichten einer künftigen Geschichtswissenschaft, Bd. II: Revolution ein historischer Längsschnitt (München 1974) 34-53; Klaus Bringmann, Das Problem einer „Römischen Revolution“, in: GWU 31 (1980) 354-377 (= ders., Ausgewählte Schriften, hg. von Jörn Kobes, Peter Scholz [Frankfurt 2001] 201-223; Rilinger, Interpretation (wie Anm.3) 279ff. mit weiteren Nachweisen (= ders., Ordo [wie Anm. 3] 123ff.).

35 Grundlegend bleibt William V. Harris, War and Imperialism in Republican Rome, 327-70 B.C. (Oxford 1979, ND 1985, 1992) und dazu etwa John North, The Development of Roman Imperialism, in: JRS 71 (1981) 1-9, sowie die einschlägigen Beiträge in: The Imperialism of Mid-Republican Rome, ed. by William V. Harris (Rom 1984), und neuerdings in: Jane Webster, Nicholas J. Cooper (Hgg.), Roman Imperialism: Post-Colonial Perspectives (Leicester 1996); s. ferner John Rich, Fear, Greed and Glory: the Causes of Roman War-Making in the Middle Republic, in: John Rich, Graham Shipley (Hgg.), War and Society in the Roman World (London 1993) 38-68; Kurt A. Raaflaub, Born to be Wolves? Origins of Roman Imperialism, in: Transitions to Empire (wie Anm. 13) 273-314, und zuletzt Armin Eich, Peter Eich, War and State-Building in Roman Republican Times, in: SCI 24 (2005) 1-33, hier 4ff.; Arthur M. Eckstein, Conceptualizing Roman Imperial Expansion under the Republic and Imperium: An Introduction, in: Companion to the Roman Republic (wie Anm. 12) 567-589; ders., Mediterranean Anarchy, Interstate War, and the Rise of Rome (Berkeley etc. 2006) $181 \mathrm{ff}$. and passim. 
sich, daß die Dichotomie von Ereignis- und Strukturgeschichte nicht nur überholt, sondern auch auf besondere Weise der konkreten Sache nicht angemessen ist: Äußere Expansion (als Ereignisgeschichte par excellence) einerseits und Integration und Konsolidierung im Inneren, gesellschaftliche Stabilisierung, ideologische Orientierung und Legitimität eines besonderen oligarchischen Regimes andererseits bedingen sich gegenseitig - die Grundeinsicht dieser Verschränkung, ihrer Komplexität und vor allem ihrer inhärenten Dynamik, die aus der Prekarität und Labilität der relativen Gewichtung der einzelnen Faktoren resultierte, dürfen wir heute (so meine ich) zu der erwähnten Basis des Erkenntnisstandes rechnen.

Auch die Frage nach der inneren ,Architektur' der res publica richtet sich längst nicht mehr nur auf die bloße Überwindung einer sterilen Erstarrung, in der sich die republikanische Geschichte als bloße ,Geschichte der herrschenden Klasse" erschöpft zu haben schien. Dafür hat John North den (im anglo-amerikanischen Raum gern plakativ zitierten) Begriff der „frozen-waste theory“ geprägt ${ }^{36}$ und damit vor allem jene kurzschlüssige Variante einer sogenannten Prosopographie zu charakterisieren versucht, die ausschließlich auf die Rekonstruktion von dynastischen Familienverbindungen, Faktionen oder ,Adelsparteien' zielte und dabei deren Existenz immer schon selbstverständlich voraussetzte. Allerdings war diese Art der ,Geschichte der herrschenden Klasse' zu diesem Zeitpunkt eigentlich schon erledigt ${ }^{37}$ - in England und Amerika hatte man nur noch nicht zur Kenntnis genommen, daß etwa in Frankreich und ausgerechnet im angeblichen Ursprungsund Heimatland dieser vorgestrigen Prosopographie, nämlich in Deutschland, die Debatte auch aus diesem speziellen Ghetto bereits ausgebrochen war ${ }^{38}$.

$\mathrm{Zu}$ diesem Zeitpunkt waren längst die ersten Versuche unternommen worden, die gesamte Breite jener soziopolitischen Strukturen inhaltlich und vor allem auch begrifflich genauer zu fassen, in die Politik, politische Institutionen und das konkrete Handeln in ihnen eingebettet waren. Mit seinem Projekt einer „politischen Grammatik" der Republik brachte Christian Meier die diesbezüglichen verstreuten Überlegungen und schüchternen Ansätze erstmals auf einen eingängigen Begriff, der sich als solcher allerdings nicht recht durchgesetzt hat - auch wenn das Konzept aus der Rückschau wie ein Vorgriff auf die Metapher des ,Textes' und der ,Textualität ${ }^{`}$ und auf die Semantik der ,Syntax anmutet, die bekanntlich den Jargon des, linguistic turn' durchziehen. Aber hier geht es um die Sache - und zwar eine Sache, der mit der Bemühung um Trennschärfe der Begrifflichkeit noch stets

\footnotetext{
36 John North, Democratic Politics in Republican Rome, in: P\&P 126 (1990) 3-21, hier 6f.; ders., Politics and Aristocracy in the Roman Republic, in: CPh 85 (1990) 277-287, hier $277 \mathrm{ff}$.

${ }^{37} \mathrm{Vgl}$. dazu grundlegend Meier, Res publica amissa (wie Anm.3) XXXIIff; 182ff. u.ö.; dens., Alltag des Historikers (wie Anm.3) 39ff. und danach Hölkeskamp, Nobilität (wie Anm.22) 41 ff.; ders., Conquest, Competition and Consensus (wie Anm.24) 13ff. (= ders., Senatvs Popvlvsqve Romanvs [wie Anm. 16] 12ff.) und zuletzt ders., Fact(ions) or Fiction? Friedrich Münzer and the Aristocracy of the Roman Republic - then and now, in: IJCT 8.1, 2001 (2002) 92-105.

$38 \mathrm{Vgl}$. William V. Harris, On Defining the Political Culture of the Roman Republic, in: CPh 85 (1990) 288-294, hier 291 zu einer selektiven Wahrnehmung der neueren anglophonen Forschung, der er treffend als „curiously insular" charakterisiert.
} 
am besten gedient ist. Und diese Sache, die das Konzept der „politischen Grammatik" bezeichnen sollte, war damit als Aufgabe sui generis programmatisch definiert und geradezu als moderne Herausforderung formuliert: Von nun an ging es um eine wirkliche, also umfassend angelegte ,Strukturgeschichte‘ jenseits der traditionellen doppelten Einengung durch eine überholte ,Verfassungsgeschichte“ einerseits und durch die ebenfalls längst angestaubten ,orthodoxen“ Dogmen einer „frozen-waste theory“, die schlicht die Vorstellung einer von selbst stabilen, in sich ruhenden Adelsrepublik und eines dementsprechenden Spektrums von arcana imperii voraussetzte, andererseits. Damit ging es nicht mehr nur um einen weiteren Versuch, endlich dem , langen Schatten' Theodor Mommsens zu entkommen und das strenge ,System“ seines „Römisches Staatsrechtes“ einfach zu einer ,Geschichte ${ }^{`}$ der politischen Institutionen und Verfahren zu historisieren ${ }^{39}$; und es ging auch nicht mehr um die empirische Widerlegung jener axiomatischen Annahmen und zirkulären Argumentationsketten, die schlicht Verwandtschafts- und andere persönliche Beziehungen aller Art zugleich als Voraussetzung, Begründung und Beleg der Konstituierung politischer Gruppierungen in Form von stabilen, über Generationen gemeinsam im Spiel um die ,Macht' agierenden ,Adelsparteien“ oder ,Faktionen' setzten ${ }^{40}$ und damit von vornherein bereits als existent nahmen, was eigentlich erst hätte erwiesen werden müssen, nämlich eine spezifische, regelmäßige und geradezu der Natur dieser Oligarchie entsprechende Verparteilichung der gesamten republikanischen Politik.

Vielmehr ging es jetzt um einen umfassend-integrativen Ansatz, der die verschiedenen Dimensionen des Politischen und seines kulturspezifischen Charakters in ihren komplexen Wechselbeziehungen erkennbar und beschreibbar machen sollte - also das spezifisch römisch-republikanische Aufeinanderbezogensein von Institutionen, Verfahren und ,Praxis' des Handelns in ihnen und durch sie einerseits und von gesellschaftlichen Kontexten und kulturellen Bedingungen andererseits, in welche die normativen Strukturen und die ,reale' Politik eingebunden waren und welche die (wechselnden, sich erweiternden und/oder verengenden) Spielräume dieses Handelns und damit auch die Grenzen dieser Spielräume bestimmten; und es ging deshalb zwangsläufig zugleich auch um die Identität(en) der darin handelnden Gruppen, ihre handlungsdeterminierenden Orientierungen und Horizonte, ihre (genutzten oder verfallenen) Chancen, Optionen und wiederum die Grenzen des Denk- und Vorstellbaren.

\footnotetext{
39 Vgl. zur Wirkungs- und Rezeptionsgeschichte des „Staatsrechts“ zuletzt Wilfried Nippel, Das Staatsrecht in der Diskussion von 1871 bis heute, in: ders., Bernd Seidensticker (Hgg.), Theodor Mommsens langer Schatten. Das römische Staatsrecht als bleibende Herausforderung für die Forschung (Hildesheim 2005) 9-60. S. außerdem zu den Potentialen und Perspektiven des „Staatsrechts“ für die weitere Forschung zur (späten) Republik Karl-Joachim Hölkeskamp, Ein „Gegensatz von Form und Inhalt“. Theodor Mommsens Konzept des republikanischen „Senatsregiments“ - Hindernis oder Herausforderung?, in: ebd. 87-129; Martin Jehne, Die Volksversammlungen in Mommsens Staatsrecht oder: Mommsen als Gesetzgeber, in: ebd. 131-160.

40 Vgl. die Angaben in Anm.37, sowie Brunt, Fall of the Roman Republic (wie Anm.14) 443-502; Meier, Alltag des Historikers (wie Anm.3) 40; 43.
} 
Im Jahre 1976, ein Jahrzehnt nach „Res publica amissa“, veröffentlichte Claude Nicolet - der sich bezeichnender- und/oder paradoxerweise zunächst als souveräner Player einer (notabene solide sozialgeschichtlich orientierten) prosopographischen Eliteforschung einen Namen gemacht hatte ${ }^{41}$ - ein Buch, das erst mittel- und langfristig, ja eigentlich nur aus der Rückschau als das erkannt wurde, was es war: Mit „Le métier de citoyen“ (oder: „The World of the Citizen“) im republikanischen $\operatorname{Rom}^{42}$ lag nun der erste Versuch einer synthetisch-vernetzten Gesamtdarstellung der soziopolitischen Ordnung der Republik vor, die gezielt und in dieser Dichte erstmals politische Institutionen und gesellschaftliche Strukturen, formalisierte Verfahren und das weitere Spektrum kultureller Praktiken ein- und zugleich aufeinanderbeziehen wollte - anders gesagt: Es war eine erste ,dichte Beschreibung' der römisch-republikanischen politischen Kultur avant la lettre.

In der Folgezeit wurden dann nicht nur die allgemeinen Anregungen aus den erwähnten Theoriedebatten der Geschichtswissenschaften wirksam, sondern auch die ebenso wichtigen, ganz konkreten Impulse aus den altertumswissenschaftlichen Nachbardisziplinen: Eine moderne Klassische Archäologie, die Monumente, Bilder und ihre Botschaften in ihren soziokulturellen Kontexten und Wirkungen zu verstehen begann, entwickelte sich ja gerade an der Repräsentationskunst, der Architektur und der urbanistischen Entwicklung des mittel- und spätrepublikanischen bzw. frühkaiserzeitlichen Rom $^{43}$. Genau damit erschloß sie einer kultur-

41 Claude Nicolet, L'ordre équestre à l'époque républicaine (312-43 av. J.-C.), Bd. I-II (Paris 1966, 1974); ders., Prosopographie et histoire sociale: Rome et l'Italie à l'époque républicaine, in: AESC 25 (1970) 1209-1228; ders., Les classes dirigeantes romaines sous la République. Ordre sénatorial et ordre équestre, in: AESC 32 (1977) 726-755.

${ }^{42}$ Le métier de citoyen dans la Rome républicaine (Paris 1976) = The World of the Citizen in Republican Rome (Berkeley etc. 1980).

43 Grundlegend sind Tonio Hölscher, Die Anfänge römischer Repräsentationskunst, in: MDAI (R) 85 (1978) 315-357; ders., Die Geschichtsauffassung in der römischen Repräsentationskunst, in: JDAI 95 (1980) 265-321; ders., Staatsdenkmal und Publikum. Vom Untergang der Republik zur Festigung des Kaisertums in Rom (Konstanz 1984); ders., Römische Bildsprache als semantisches System (Heidelberg 1987, erweiterte engl. Ausgabe: The Language of Images in Roman Art [Cambridge 2004]); ders., Römische Nobiles und hellenistische Herrscher, in: Akten des XIII. Internationalen Kongresses für Klassische Archäologie, Berlin 1988 (Mainz 1990) 73-84; ders., Bilderwelt, Formensystem, Lebenskultur. Zur Methode archäologischer Kulturanalyse, in: SIFC 3a ser. 10 (1992) 460-484; ders., Die Alten vor Augen. Politische Denkmäler und öffentliches Gedächtnis im republikanischen Rom, in: Gerd Melville (Hg.), Institutionalität und Symbolisierung. Verstetigungen kultureller Ordnungsmuster in Vergangenheit und Gegenwart (Köln etc. 2001) 183-211; ders., Augustus und die Macht der Archäologie, in: La révolution romaine après Ronald Syme (wie Anm.2) 237-281; ders., Images of War in Greece and Rome: Between Military Practice, Public Memory, and Cultural Symbolism, in: JRS 93 (2003) 1-17; ders., The Transformation of Victory into Power: From Event to Structure, in: Sheila Dillon, Katherine E. Welch (Hgg.), Representations of War in Ancient Rome (Cambridge 2006) 27-48; Paul Zanker, Augustus und die Macht der Bilder (München 1987 u. ö.) und jetzt die knappen Überblicke in Adolf H. Borbein, Tonio Hölscher, Paul Zanker, Klassische Archäologie. Eine Einführung (Berlin 2000), sowie Karl-Joachim Hölkeskamp, Images of Power: Memory, Myth and Monuments in the Roman Republic (Rezensionsartikel zu dem ebenfalls wichtigen Buch von Peter J. Holliday, The Origins of Roman Historical Commemoration in the Visual Arts [Cambridge 2002]), in: SCI 24 (2005) 249-271; Ann 
historisch gewendeten Althistorie nicht nur ganz neue, eben auch historisch zu verstehende und auszuwertende Quellengattungen, sondern bot gleichzeitig auch die methodischen Ansätze an, diese materielle Hinterlassenschaft als Zeugnisse im Sinne einer neuen Strukturgeschichte zu ,lesen' - nämlich als Umsetzung von kollektiven Werten, Ansprüchen und Zielen in visuelle Medien öffentlicher Selbstdarstellung, in monumentale Architektur und andere Formen einer repräsentativen Kunst, die Ideologien und kollektives Selbstverständnis in eindringlichen Bildern der Macht, der imperialen Größe und des Glanzes sichtbar werden ließ und dabei zugleich Zustimmung und Identifizierung des Betrachters einforderte. Auch wenn noch längst nicht alle Defizite an Begriffen, Theorien, Methoden, Modellen überwunden sind; auch wenn wir hier längst noch nicht das Ende der Möglichkeiten und Potentiale erkennen können, und selbst wenn wir nicht einmal den Anfang dieses Endes, sondern allenfalls das Ende eines (immerhin vielversprechenden) Anfangs erreicht haben sollten ${ }^{44}$ : Das Angebot der Archäologie war (und ist) ein qualitativer Sprung in der erwähnten „Verfeinerung des Registers der Geschichtsschreibung“ - ob das etwa schon als (Effekt oder Ausläufer des), visual' oder ,iconic turn“ bezeichnet werden kann ${ }^{45}$, kann man hier getrost dahingestellt sein lassen.

Eine Integration dieser Ansätze und Impulse erscheint vor allem auf der Basis eines historisch-politikwissenschaftlich fundierten Konzeptes der ,politischen Kultur' möglich, unter dessen Dach die verschiedenen oben dargelegten Zugänge und methodischen Ansätze, Daten und ihre Deutungen kontrolliert und fruchtbar in einen neuen, differenzierten Zusammenhang gebracht werden können. Diese Debatte hat gleich mehrere Ebenen: Da ist zunächst die - zugegebenermaßen längst überfällige und notwendige - Diskussion über die Beziehungen zwischen Elite und Volk, die Funktionen der Institutionen und Verfahren politischen Entscheidens und damit über die Frage nach Möglichkeiten und Grenzen formaler Partizipation an Politik: Diese von Fergus Millar zwar angestoßene, aber nie aktivdiskursiv betriebene Debatte ist (vor allem dank seiner Kritiker) bis heute nicht beendet ${ }^{46}$ - und das ist auch gut so, droht doch die reduktionistisch-formalistische

L. Kuttner, Roman Art during the Republic, in: Harriet I. Flower (Hg.), The Cambridge Companion to the Roman Republic (Cambridge 2004) 294-321; Katherine E. Welch, Art and Architecture in the Roman Republic, in: Companion to the Roman Republic (wie Anm.12) 478-542.

${ }^{44}$ Damit versuche ich den diesbezüglichen eher skeptischen Zwischenbilanzen einen gewissermaßen optimistischen ,turn' zu geben: Tonio Hölscher, Klassische Archäologie am Ende des 20. Jahrhunderts. Tendenzen, Defizite, Illusionen, in: Die Wissenschaften vom Altertum (wie Anm. 28) 197-228; ders., Vorläufige Überlegungen zum Verhältnis von Theoriebildung und Lebenserfahrung in der Klassischen Archäologie, in: Stefan Altekamp, Mathias René Hofter, Michael Krumme (Hgg.), Posthumanistische Klassische Archäologie. Historizität und Wissenschaftlichkeit von Interessen und Methoden (München 2001) 173-187. Vgl. auch die allgemeinen Überlegungen zur (eben auch politischen) „Macht der Denkmäler" in Hölschers Beitrag in diesem Band

45 Vgl. dazu etwa Bachmann-Medick, Cultural Turns (wie Anm.30) 329ff.; Tschopp, Weber, Grundfragen (wie Anm.30) 99ff., jeweils mit weiteren Nachweisen.

46 Vgl. den in Anm. 10 genannten Aufsatz Fergus Millars, sowie ders., Politics, Persuasion, and the People before the Social War (150-90 B.C.), in: JRS 76 (1986) 1-11; ders., Political Power in MidRepublican-Rome: Curia or Comitium?, in: JRS 79 (1989) 138-150; ders., Popular Politics at Rome in the Late Republic, in: Irad Malkin, Zeev W. Rubinsohn (Hgg.), Leaders and Masses in 
plakative Deklaration der Republik zu einer Form der ,direkten Demokratiec durch Millar in nochmals vereinfachter Form zu einer neuen Orthodoxie zu gerinnen (übrigens wiederum in erster Linie in England, wo die Kritik gelegentlich geradezu systematisch ignoriert wird $)^{47}$. Darüber sind wir eigentlich schon seit den frühen 90er Jahren hinaus. In dieser Debatte geht es mittlerweile auch und vor allem darum, die eigentlichen Fundamente der politischen Kultur im engeren Sinne zu bestimmen - einer genuin ,republikanischen' Kultur, die auf breiter Zustimmung beruhte und sich (auch deswegen) durch hohe Flexibilität, Anpassungs- und Entwicklungsfähigkeit auszeichnete.

Es geht also heute darum, die Voraussetzungen und Grundbedingungen, gewissermaßen die Konditionen und die Konditionierungen von Politik als institutionalisiertem Entscheidungshandeln schärfer in den Blick zu nehmen: Man fragt jetzt nach Weltbildern und Wertvorstellungen, nach Orientierungswissen, Wahrnehmungsweisen und Deutungsmustern, die tief verwurzelt sind und daher selbst gar nicht zum Gegenstand von ,Politik‘ werden (können), die aber sehr wohl die Einstellungen zu ,Politik' und Krieg, etwa auch zu Eroberung und Expansion, bestimmen. Darüber hinaus begründen solche gängigen Einstellungen und allgemein unstrittigen Überzeugungen die Anforderungen an politische Führung und die Erwartungen an ganze politische Klassen und ihre Fähigkeiten. Umgekehrt beruhen darauf jene Verhaltenscodes, die wiederum die Art und Weise der Erfüllung solcher Erwartungen durch die politisch Handelnden selbst steuern: Ihre Rollen und Verhaltensmuster und damit die ,Dramaturgie‘ politischen Handelns, Formen und Medien der Selbstverständigung und Selbstdarstellung und die Art und Weise des öffentlichen Auftretens der politischen Klasse und ihres Umgangs mit dem Volk werden dadurch letztlich geprägt - mithin das ganze kulturspezifische Spektrum der ,Politik des Pomps', der Spiele und anderen Spektakel ${ }^{48}$. Vor allem dieser

the Roman World. Studies in Honor of Zvi Yavetz (Leiden 1995) 91-113; (= ders., Rome [wie Anm. 10] 143-161 bzw. 85-108; 91-103); ders., The Crowd in Rome in the Late Republic (Ann Arbor 1998). S. dazu Martin Jehne, Einführung: Zur Debatte um die Rolle des Volkes in der römischen Politik, in: Martin Jehne (Hg.), Demokratie in Rom? Die Rolle des Volkes in der Politik der römischen Republik (Stuttgart 1995) 1-9; John North, Pursuing Democracy, in: Alan K. Bowman, Hannah M. Cotton et al. (Hgg.), Representations of Empire. Rome and the Mediterranean World (Oxford 2002) 1-12; Allen M. Ward, How Democratic was the Roman Republic?, in: NECJ 31.2 (2004) 101-119; Robert Morstein-Marx, Mass Oratory and Political Power in the Late Roman Republic (Cambridge 2004) 1ff.; Karl-Joachim Hölkeskamp, The Roman Republic: Government of the People, by the People, for the People? (Rezensionsartikel zu Millar, Crowd), in: SCI 19 (2000) 203-223 (erweiterte deutsche Fassung in: ders., Senatvs Popvlvsqve Romanvs [wie Anm. 16] 257-280); ders., Rekonstruktionen (wie Anm. 11) 9ff.

47 Vgl. dazu Hölkeskamp, Rekonstruktionen (wie Anm.11) 16f. mit Nachweisen.

48 Vgl. dazu einstweilen Florence Dupont, L'acteur-roi ou le théâtre dans la Rome antique (Paris 1985) $17 \mathrm{ff}$. und passim; Bettina Bergmann, Introduction, in: Bettina Bergmann, Christina Kondoleon (Hgg.), The Art of Ancient Spectacle (New Haven etc. 1999) 9-35 und die einschlägigen Beiträge in diesem Band; Richard C. Beacham, Spectacle Entertainments of Early Imperial Rome (New Haven etc. 1999); Egon Flaig, Ritualisierte Politik. Zeichen, Gesten und Herrschaft im Alten Rom (Göttingen 2003); Andrew Bell, Spectacular Power in the Greek and Roman City (Oxford 2004); Harriet I. Flower, Spectacle and Political Culture in the Roman Republic, in: Cam- 
Einsicht - und den wichtigen konkreten Anregungen durch die empirischen Studien zu den ,civic rituals` der Stadtstaatskulturen im Italien des Spätmittelalters und der Renaissance ${ }^{49}$ und wohl auch der allgemeinen Inspiration durch eine weitere Wende, den vieldiskutierten ,performative $\operatorname{turn}^{60}$ - verdankt sich das neue intensive Interesse an jenen „symbolisch-expressiven“ Ritualen und Zeremonien, die für die politische Kultur der Republik (und dann auch der Kaiserzeit) so typisch und vielsagend sind: Dazu gehört natürlich einerseits der (im doppelten Sinne) besonders ,spektakuläre' Triumph ${ }^{51}$ und andererseits das eigentümliche Ritual der pompa funebris mit ihrer dicht vernetzten Syntax aus vielschichtigen und vieldeutigen Symbolen, Bildern und Botschaften ${ }^{52}$ - und nicht zuletzt auch die bezeichnenden, gewissermaßen absichtsvoll ,transgressiven“ Variierungen gerade dieses Rituals seit der ,letzten Generation‘ der Republik und dann (ganz gezielt)

bridge Companion to the Roman Republic (wie Anm.43) 322-343; Geoffrey S. Sumi, Ceremony and Power. Performing Politics in Rome between Republic and Empire (Ann Arbor 2005); Hans Beck, Züge in die Ewigkeit. Prozessionen durch das republikanische Rom, in: GFA 8 (2005) 73104; Bernhard Linke, Politik und Inszenierung in der Römischen Republik, in: Aus Politik und Zeitgeschichte 7 (2006) 33-38; Karl-Joachim Hölkeskamp, Rituali e cerimonie „alla romana“. Nuove prospettive sulla cultura politica dell'età repubblicana, in: StudStor 47 (2006) 319-363; ders., Pomp und Prozessionen. Rituale und Zeremonien in der politischen Kultur der römischen Republik, in: Jahrbuch des Historischen Kollegs 2006 (erschienen 2007) 35-72; ders., Hierarchie und Konsens. Pompae in der politischen Kultur der römischen Republik, in: Alexander Arweiler (Hg.), Machtfragen. Zur kulturellen Repräsentation und Konstruktion von Macht (Stuttgart 2008) 79-126.

49 Vgl. Edward Muir, Civic Rituals in Renaissance Venice (Princeton 1981); ders., Ritual in Early Modern Europe (Cambridge 1997) hier bes. 232ff.; ders., Representations of power, in: John M. Najemy (Hg.), Italy in the Age of the Renaissance 1300-1550 (Oxford 2004) 226-245; Richard C. Trexler, Public Life in Renaissance Florence (Ithaca etc. 1980), sowie die gerade wegen ihrer komparativen Ansätze wichtigen Sammelbände von Anthony Molho, Kurt Raaflaub, Julia Emlen (Hgg.), City-States in Classical Antiquity and Medieval Italy (Stuttgart 1991); Mogens Herman Hansen (Hg.), A Comparative Study of Thirty City-State Cultures (Kopenhagen 2000) und dazu Karl-Joachim Hölkeskamp, in: BMCR 2004.04.03.

50 Vgl. dazu Bachmann-Medick, Cultural Turns (wie Anm. 30) 104ff.; Tschopp, Weber, Grundfragen (wie Anm. 30) 111ff. Vgl. dazu allgemein Doris Kolesch, Rollen, Rituale und Inszenierungen, in: Handbuch der Kulturwissenschaft, Bd.2 (wie Anm. 30) 277-292.

51 Vgl. dazu außer der in Anm. 48 genannten Literatur die (recht radikale), Lesung' dieses Rituals durch Egon Flaig, Warum die Triumphe die römische Republik ruiniert haben - oder: Kann ein politisches System an zuviel Sinn zugrunde gehen?, in: Karl-Joachim Hölkeskamp, Jörn Rüsen, Elke Stein-Hölkeskamp, Heinrich Th. Grütter (Hgg.), Sinn (in) der Antike. Orientierungssysteme, Leitbilder und Wertkonzepte im Altertum (Mainz 2003) 299-313; s. dagegen Tanja Itgenshorst, Tota illa pompa. Der Triumph in der römischen Republik (Göttingen 2005); Richard Brilliant, „Let the Trumpets Roar!“ The Roman Triumph, in: The Art of Ancient Spectacle (wie Anm. 48) 221-229; Karl-Joachim Hölkeskamp, Der Triumph - „erinnere Dich, daß Du ein Mensch bist“, in: ders., Elke Stein-Hölkeskamp (Hgg.), Erinnerungsorte der Antike. Die römische Welt (München 2006) 258-276; 745-747, und zuletzt Mary Beard, The Roman Triumph (Cambridge, Mass. etc. 2007).

52 Vgl. dazu Harriet Flower, Ancestor Masks and Aristocratic Power in Roman Culture (Oxford 1996); dies., Der Leichenzug - die Ahnen kommen wieder, in: Erinnerungsorte der Antike (wie Anm. 51) 321-337; 752-753, sowie John Bodel, Death on Display: Looking at Roman Funerals, in: The Art of Ancient Spectacle (wie Anm. 48) 259-281. 
durch Augustus ${ }^{53}$. Aus der erwähnten Einsicht resultiert schließlich auch noch eine erweiterte, differenzierte und mehrdimensionale Sicht der eben auch nur oberflächlich bzw. scheinbar ausschließlich zweckrationalen „technisch-instrumentellen" ${ }^{44}$ Verfahren der formalen Entscheidungsfindung in den politischen Institutionen, etwa der eigentümlichen Dramaturgie der Debatten in den contiones respektive der Wahlen und sonstigen Abstimmungen in den Comitien ${ }^{55}$.

Allgemeiner formuliert: Eine ,politische Kultur ' hat also nicht nur eine „Inhaltsseite“, sondern auch eine "Ausdrucksseite“ und eine entsprechende „kognitive“ Ebene $^{56}$ : Sie hat zeremonielle und rituelle, performative, symbolische und auch ästhetische Dimensionen, die für die permanente Reproduktion der Legitimität der Elite und des politischen Systems insgesamt konstitutiv sind; denn es ist vor allem diese „Ausdrucksseite“, die der Erzeugung von Zugehörigkeit und Zustimmung, der Stiftung von Sinn und Sinnhaftigkeit politischen Handelns und damit der Begründung einer kollektiven Identität eines ganzen Volkes und seiner politischen Klasse dient. Noch einmal allgemeiner formuliert: Dieser neue Blick auf die (im doppelten Sinne) kulturelle Einbettung des Politischen als „Medium der

53 Vgl. dazu den Beitrag von Egon Flaig in diesem Band.

54 Vgl. zu diesen Begriffen (und der Problematik der Dichotomie ,Ritual/Zeremonie‘-,Verfahren') Barbara Stollberg-Rilinger, Einleitung, in: dies. (Hg.), Vormoderne politische Verfahren (Berlin 2001) 9-24, hier 12; 18; 20 u. ö.; vgl. dies., Zeremoniell, Ritual, Symbol. Neue Forschungen zur symbolischen Kommunikation in Spätmittelalter und Früher Neuzeit, in: ZHF 27 (2000) 389-405, hier 390ff.; Uwe Goppold, Politische Kommunikation in den Städten der Vormoderne. Zürich und Münster im Vergleich (Köln etc. 2007) $31 \mathrm{ff.;} 34 \mathrm{ff}$.

55 Vgl. dazu generell Hölkeskamp, Rekonstruktionen (wie Anm.11) 88f. S. zu den Contionen etwa dens., Oratoris maxima scaena: Reden vor dem Volk in der politischen Kultur der Republik, in: Demokratie in Rom? (wie Anm.46) 11-49 (= Hölkeskamp, Senatvs Popvlvsqve Romanvs [wie Anm.16] 219-256, mit Ergänzungen, hier 234ff.); Morstein-Marx, Mass Oratory (wie Anm. 46) 7ff.; 34ff.; 154ff.; 256ff. u.ö.; Frank Bücher, Verargumentierte Geschichte. Exempla Romana im politischen Diskurs der späten römischen Republik (Stuttgart 2006) 29ff., sowie bereits Francesco Pina Polo, Contra arma verbis. Der Redner vor dem Volk in der späten römischen Republik (Stuttgart 1996) und die nach wie vor nicht ersetzte Materialsammlung dess., Las contiones civiles y militares en Roma (Zaragoza 1989) $244 \mathrm{ff}$. S. zur „expressiv-symbolischen“ Funktion der Comitien grundlegend Martin Jebne, Integrationsrituale in der römischen Republik. Zur einbindenden Wirkung der Volksversammlungen, in: Sinn (in) der Antike (wie Anm. 51) 279-297.

56 Vgl. zu diesen Konzepten Karl Robe, Politische Kultur und ihre Analyse. Probleme und Perspektiven der politischen Kulturforschung, in: HZ 250 (1990) 321-346; vgl. dazu und zum folgenden Hölkeskamp, Rekonstruktionen (wie Anm.11) 58ff. Vgl. generell zu den „symbol“- bzw. „wissenszentrierten“ Ansätzen der neueren Politischen Kulturforschung etwa Birgit Schwelling, Politische Kulturforschung als kultureller Blick auf das Politische. Überlegungen zu einer Neuorientierung der Politischen Kulturforschung nach dem „cultural turn“, in: Zschr. für Politikwissenschaft 11,2 (2001) 601-629; Carola Lipp, Politische Kultur oder das Politische und Gesellschaftliche in der Kultur, in: Kulturgeschichte Heute (wie Anm.29) 78-110; Thomas Mergel, Überlegungen zu einer Kulturgeschichte der Politik, in: GuG 28 (2002) 574-606, hier 583ff.; Achim Landwehr, Diskurs - Macht - Wissen. Perspektiven einer Kulturgeschichte des Politischen, in: AKG 85 (2003) 71-117; Luise Schorn-Schütte, Historische Politikforschung. Eine Einführung (München 2006) 67ff. 
Macht “ 57 ist wiederum integraler Bestandteil eines umfassenden Paradigmenwechsels, der gewissermaßen im Gewand eines weiteren ,turn' daherkommt, nämlich in einer „,kommunikationstheoretischen Wende“ in der Wahrnehmung historischer Gegenstände“, die keineswegs nur in der Neueren und Neuesten (Politik-)Geschichte in den Mittelpunkt des Interesses rückt: Danach stellen sich Politik generell und das konkrete Handeln individueller oder kollektiver Akteure in politicis eben nicht mehr als „eindimensionaler Akt oder Prozess“ dar, „in dem von oben nach unten dekretiert, regiert, entschieden“ werde - vielmehr sei „politisches Handeln immer kommunikatives Handeln“, das mithin „Partizipation“ der Adressaten voraussetze (wenn auch „nicht unbedingt im Sinne einer gleichberechtigten Teilhabe“): In dieser Perspektive erscheint Politik als „Kommunikationszusammenhang“, „der in seiner Form symbolisch vermittelt und damit auch entscheidend von den medialen Möglichkeiten einer Gesellschaft“ konditioniert und determiniert sei, nämlich von dem gesamten kulturspezifischen „Arsenal kommunikativer Formen und Instrumente“ 58 von der politischen Rede über die Rituale bis zu den dafür reservierten Räumen.

\section{Kommunikation, Interaktion und vormoderne ,Stadtstaatlichkeit': Anregungen und Ansätze der historischen Politikforschung}

In den letzten Jahren sind erst die Grundlinien der eigentümlich komplexen politischen Kultur der römischen Republik beschrieben worden - eine umfassende Gesamtdarstellung steht dagegen bisher aus. Immerhin lassen sich einige wichtige Koordinaten bzw. Dimensionen namhaft machen: Da ist zunächst jene besondere Kombination - oder genauer: Komplementarität - des allgegenwärtigen Systemprinzips der steilen Hierarchie einerseits und des auffälligen Grades der gesellschaftlichen Homogenität und Kohärenz andererseits, die erst einmal analysiert und erklärt sein will - anders formuliert, diesmal mit Egon Flaig: Die oft diagnostizierte eigentümliche „Gehorsamstiefe“ der breiten Schichten des populus Romanus darf nicht einfach vorausgesetzt werden - sie ist vielmehr als eine wertvolle, seit dem 2.Jahrhundert v. Chr. womöglich allmählich knapper werdende Ressour-

${ }^{57}$ Ich wandle hier die programmatisch-knappe Charakterisierung der Macht als „Medium des Politischen“ ab: Rudolf Schlögl, Politik- und Verfassungsgeschichte, in: Joachim Eibach, Günther Lottes (Hgg.), Kompass der Geschichtswissenschaften. Ein Handbuch (Göttingen 2002) 95-111, hier 104.

58 Vgl. dazu Schlögl, Politik- und Verfassungsgeschichte (wie Anm. 57) 109; Ute Frevert, Neue Politikgeschichte, in: Kompass der Geschichtswissenschaften (wie Anm. 57) 152-164, hier 158 und 159 (Zitate); dies., Neue Politikgeschichte: Konzepte und Herausforderungen, in: dies., HeinzGerhard Haupt (Hgg.), Neue Politikgeschichte. Perspektiven einer historischen Politikforschung (Frankfurt etc. 2005) 7-26, hier 14ff. S. generell Thomas Mergel, Kulturwissenschaft der Politik: Perspektiven und Trends, in: Friedrich Jaeger, Jörn Rüsen (Hgg.), Handbuch der Kulturwissenschaften, Bd.3: Themen und Tendenzen (Stuttgart etc. 2004) 413-425; Goppold, Politische Kommunikation (wie Anm. 54) 22ff.; $37 \mathrm{ff}$. 
ce dieses spezifischen meritokratisch-oligarchischen Regimes zu konzeptualisieren, deren Quellen bzw. Wurzeln und deren Reproduktionskapazität erst einmal genauer zu bestimmen $\operatorname{sind}^{59}$. Denn naheliegend oder gar selbstverständlich ist diese Kombination von Hierarchie und Homogenität, die diese Gesellschaft in Meiers Worten „monistisch“ machte ${ }^{60}$, eben gerade nicht: Hierarchie und Herrschaft, Macht und ihre Ausübung von oben nach unten spiegelten sich nicht nur im Gefälle von populus Romanus, Untertanen in den Provinzen und ,Bundesgenossen und Freunden' wider, sondern sie durchdrangen auch die Gesellschaft(en) Roms, Italiens und des Imperiums selbst, und sie strukturierten sogar ganz direkt und ungebrochen alle politischen Institutionen und Verfahren; und nicht zuletzt waren sie in den bereits erwähnten Zeremonien und Ritualen der Selbstdarstellung und -bestätigung vom Triumph bis zur pompa funebris und selbst noch in den Mythen, Legenden und Ideologien präsent - anders gesagt: Es gab keine Institution, die ,bürgerliche' Gleichberechtigung in politicis hätte herstellen können oder gar sollen, es gab keine gesellschaftliche Nische, in der Egalität einen sicheren, angestammten und akzeptierten Platz gehabt hätte, und es gab noch nicht einmal eine Idee, die in Gleichheit einen Wert gesehen hätte.

Ein konstitutiver Faktor war sicherlich jenes ebenso dichte wie feste Netz der Strukturen und Mechanismen, das wir herkömmlicherweise mit Begriffen wie ,Bindungswesen', Patronage und Clientel zu fassen versuchen ${ }^{61}$. Dieses komplexe Netz asymmetrisch-reziproker Beziehungen, die von etablierten und sogar ererbten Verpflichtungsverhältnissen bis hin zu lockeren und eher unverbindlichen Loyalitäten reichten, garantierte, reproduzierte und sanktionierte die vertikale wie horizontale Integration von Ständen, Gruppen und Individuen - als solches, als Struktur, zerriß dieses Netz ja nicht einmal in der Krise der Republik und überdauerte sogar ihren Untergang. Eine weitere Grundlage dieser Komplementarität bestand darin, daß die Kohärenz durch Konsens begründet und kontinuierlich bestätigt wurde, und zwar durch die besonders sorgfältige, permanente und vielgestaltige Pflege dieses Konsenses durch eine politische Klasse, die an der Spitze aller Hierarchien stand: Ihre Identität als ,Meritokratie‘ wurde durch ein reiches Repertoire von Ritualen und sonstigen symbolischen Formen der (Selbst-)Repräsentation reproduziert und geradezu immer neu konstruiert, die der Sichtbarmachung

59 Vgl. Flaig, Ritualisierte Politik (wie Anm. 48) $13 \mathrm{ff}$.

60 S. Meier, Res publica amissa (wie Anm. 3) 53; 57, vgl. XXVff.; $52 \mathrm{ff.}$

61 Vgl. dazu den Beitrag von Jean-Michel David in diesem Band und bereits dens., Le patronat judiciaire au dernier siècle de la République romaine (Rom 1992); Élizabeth Deniaux, Clientèles et pouvoir à l'époque de Cicéron (Rom 1993), und jetzt dies., Patronage, in: Companion to the Roman Republic (wie Anm.12) 401-420, sowie bereits Brunt, Fall of the Roman Republic (wie Anm.14) 382-442; Alexander Yakobson, Elections and Electioneering in Rome. A Study in the Political System of the Late Republic (Stuttgart 1999) 65ff.; $112 \mathrm{ff}$. u. ö. Zur Rolle der ,auswärtigen` Clientel s. zuletzt den Überblick von Altay Coşkun, Freundschaft und Klientelbindung in Roms auswärtigen Beziehungen. Wege und Perspektiven der Forschung, in: ders., Heinz Heinen, Manuel Tröster (Hgg.), Roms auswärtige Freunde in der späten Republik und im frühen Prinzipat (Göttingen 2005) 1-30. 
und damit dem Einsatz des kulturspezifischen ,symbolischen Kapitals‘ als ,Kredit‘ dienten ${ }^{62}$.

Diese Sichtbarmachung bzw. Sichtbarkeit ist durchaus wörtlich zu nehmen; denn die erwähnten Formen brauchten durchweg notwendig eine Verortung in den öffentlichen Räumen einer stadtstaatlichen Kultur - und auch diese ,Ver-Ortung' ist im Wortsinne zu verstehen. Denn diese Räume waren als die ,Foren“ (diesmal im doppelten Sinne des Begriffs) der direkten Interaktion und der besonders dichten „Kommunikation unter Anwesenden“63 ihrerseits unverzichtbare und unverwechselbare Bestandteile der typischen politisch-sakralen Topographie der ,Stadtstaatlichkeit ${ }^{64}$ : Die charakteristische Unmittelbarkeit dieser spezifischen „kommunikativen Form des Politischen in der vormodernen Stadt“, die Sichtbarkeit und damit die sinnliche Erfahrbarkeit aller Verfahren, Rituale und Zeremonien auch für die nur passiv Teilnehmenden gehörten in einer solchen „Kultur des unmittelbaren Handelns“, wie Rudolf Schlögl respektive Tonio Hölscher sie definiert haben ${ }^{65}$, letztlich selbst zum Repertoire der Konsensproduktion - diese „gesteigerte Aufmerksamkeit für die räumliche Seite der geschichtlichen Welt“ mag man als ,spatial turn' bezeichnen, natürlich wiederum mit den eingangs erwähnten Reserven gegenüber derlei Etiketten ${ }^{66}$ : Jedenfalls könnte diese Aufmerksamkeit durchaus eine weitere „Verfeinerung des Registers der Geschichtsschreibung“ führen - auch derjenigen über den Dictator Caesar, der nicht zufällig gerade in die politisch-sakrale Topographie des republikanischen Rom ebenso massiv wie nachhaltig eingriff ${ }^{67}$.

Schließlich kann man nicht bei einer bloßen Darstellung der „Inhalts-“ und „Ausdrucksseite“ stehenbleiben, sondern muß ihre spezifische Verschränkung, das

${ }^{62}$ Vgl. dazu zuletzt Karl-Joachim Hölkeskamp, Konsens und Konkurrenz. Die politische Kultur der römischen Republik in neuer Sicht, in: Klio 88,2 (2006) 360-396.

${ }^{63}$ Vgl. dazu grundlegend Rudolf Schlögl, Vergesellschaftung unter Anwesenden. Zur kommunikativen Form des Politischen in der vormodernen Stadt, in: ders. (Hg.), Interaktion und Herrschaft. Die Politik der frühneuzeitlichen Stadt (Konstanz 2004) 9-60, hier 21 ff.; vgl. auch Goppold, Politische Kommunikation (wie Anm.54) 30f. und passim.

${ }^{64}$ Vgl. dazu grundlegend Tonio Hölscher, Öffentliche Räume in frühen griechischen Städten (Heidelberg 1998); s. zur urbanen Topographie des republikanischen Rom etwa Christine Döbler, Politische Agitation und Öffentlichkeit in der späten Republik (Frankfurt etc. 1999) 18ff.; JeanMichel David, I luoghi della politica dalla Repubblica all'Impero, in: Andrea Giardina (Hg.), Storia di Roma dall'antichità ad oggi (Rom 2000) 57-83; Karl-Joachim Hölkeskamp, Capitol, Comitium und Forum. Öffentliche Räume, sakrale Topographie und Erinnerungslandschaften, in: Stefan Faller (Hg.), Studien zu antiken Identitäten (Würzburg 2001) 97-132 (= Hölkeskamp, Senatvs Popvlvsqve Romanvs [wie Anm.16] 137-168, mit Ergänzungen) und zuletzt die einführende ,dichte Beschreibung ' bei Bücher, Verargumentierte Geschichte (wie Anm.55) 20ff.; Morstein-Marx, Mass Oratory (wie Anm. 46) 42ff.

65 Schlögl, Vergesellschaftung unter Anwesenden (wie Anm.63) 21ff.; 46ff. und passim; Tonio Hölscher, Aus der Frühzeit der Griechen. Räume - Körper - Mythen (Stuttgart etc. 1998) 69ff.; ders., Körper, Handlung und Raum als Sinnfiguren in der griechischen Kunst und Kultur, in: Sinn (in) der Antike (wie Anm. 51) 163-192, hier 163f.; 182ff., auch zum Folgenden.

66 Schlögel, Im Raume lesen wir die Zeit (wie Anm.31) 68, vgl. 12; $60 \mathrm{ff} . ; 502$.

${ }^{67}$ Vgl. dazu den Beitrag von Giuseppe Zecchini in diesem Band. 
Aufeinanderbezogensein der formalen und sozialen, ideologischen und symbolischen Ebenen zu einem eigenen Thema machen - dabei können auch moderne institutionen- und systemtheoretische Modelle hilfreich sein, etwa wenn es um die trennscharfe Definition und Analyse dessen geht, was unter den kulturellen Konditionen der ,Stadtstaatlichkeit' eine zentrale Rolle spielte, nämlich Charakter, Status und Funktionen von ,Öffentlichkeit', die ja hier zugleich ,communauté und ,publicitée, abstrakte Bürgerschaft und konkrete Institution, Forum, Comitium und comitia, durch Monumente konkret markierter Platz und sozial konstituierter bzw. konstruierter Raum bedeutet ${ }^{68}$. Ja, man könnte eine ebenso gewagte wie etwas frivol formulierte, mit den Kernkonzepten der Theoriedebatten spielende Prognose wagen: Diese ,Foren' - oder allgemein: das idealtypische ,Forum' des öffentlichen Raums des Stadtstaates der Vormoderne als konkreter Ort der „Vergesellschaftung unter Anwesenden" - könnten thematisch wie konzeptionell als das zentrale empirische Experimentierfeld - oder eben auch als Ort der verdich-

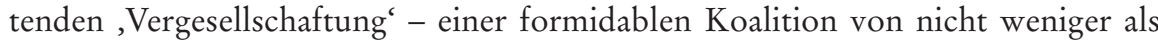
drei kulturalistischen (Teil-)Wenden dienen: des ,performative', ,spatial' und ,visual turn'.

\section{Nochmals zur ,Krise` der Republik: Paradoxie als Herausforderung}

Das ist ein ebenso faszinierendes wie ambitioniertes Programm. Derzeit steht allerdings in vieler Hinsicht die Probe aufs Exempel bezüglich der Operationalisierbarkeit der einzelnen Ansätze (oder Ansprüche), Modelle (oder Moden) noch aus - gerade wenn es um die dichte Beschreibung der Krise und des Untergangs der Republik als eines komplexen Prozesses geht, dessen zunehmende Eigendynamik einerseits von kontingenten ,Ereignissen 'vorangetrieben wurde, andererseits aber von ,systemisch' miteinander vernetzten und sich gegenseitig verstärkenden Faktoren bedingt war: Auch die dynamische Destabilisierung der Aristokratie, die durch Auftreten und Handeln außergewöhnlicher Figuren aus ihrer eigenen Mitte zugleich demonstriert und weiter angetrieben wurde, der zunächst schleichende, dann rasante Verlust an Kapazitäten der Konfliktbegrenzung und pragmatischen Problembewältigung wird so erst beschreibbar und damit verständlich - aber zugleich scheint eben genau hier bereits ein Grundproblem, ja eine Aporie des Begriffs ,Krise' als prozessualer Kategorie einerseits und einer systemtheoretisch inspirierten Modellbildung andererseits auf, nämlich das Problem des Verortens oder ,Einschreibens' einer Prozessualität und ihrer Dynamik in ein ,systemisches` Raster. In unserem Fall heißt das konkret: Wir wissen ja mittlerweile sehr wohl, daß unsere common sense-Konzeption der ,Krise der Republik' genau an diesem

${ }^{68}$ Vgl. dazu Karl-Joachim Hölkeskamp, Institutionalisierung durch Verortung. Die Entstehung der Öffentlichkeit im frühen Griechenland, in: Sinn (in) der Antike (wie Anm. 51) 81-104, hier $85 \mathrm{ff} . ;$ ders., Rekonstruktionen (wie Anm.11) $70 \mathrm{ff}$. 
Problem leidet, daß sie sich (zumindest implizit und tendenziell notwendig) auf ein statisches Gegen- oder (im doppelten Sinne) Vor-Bild einer in sich selbst ruhenden, mittleren oder ,klassischen' Republik bezieht - gerade das zuletzt genannte Etikett für den Zeitraum von etwa 300 (oder auch präziser: 287 als ,Ende der Ständekämpfe`) bis zum „erneut revolutionären“ Tribunat des Ti. Gracchus im Jahre $133^{69}$ ist in dieser Hinsicht sehr bezeichnend. Wir sind uns in den letzten Jahren immer bewußter geworden, daß es dieses ,goldene Zeitalter der Oligarchie ${ }^{6}$ aber nie gegeben hat. Denn durch die Konstitution der politischen Klasse selbst und schon seit ihrer Entstehung aus dem Geist von „conquest and competition“ hatten der „consensus“ und die „monistische“ Homogenität immer einen schweren $\operatorname{Stand}^{70}$ : Der konstitutiven Verschränkung von Expansion und Integration war ein äußerst dynamisches, ja explosives Potential an krisenhafter Konfliktträchtigkeit inhärent, das aus der erwähnten Prekarität und Labilität des immer neu auszutarierenden Gleichgewichts zwischen individueller Konkurrenz und kollektivem Konsens, aristokratisch-anarchischer Agonalität und meritokratisch-disziplinierter Loyalität resultierte - und zwar von Anfang an, also seit der erwähnten Phase der Genese dieser Klasse im 4. und frühen 3. Jahrhundert. Und auch schon von Anfang an mußte die deswegen in sich und aus sich selbst heraus permanent bedrohte Elite diese Aporie aushalten, indem sie die zahllosen und letztlich unvermeidlichen praktischen Kollisionen zwischen Konsens und Konkurrenz mit der ebenfalls schon erwähnten Kapazität zu einer geradezu permanenten pragmatischen Regulierung der Konflikte und ihr selbst inhärenten Spannungen einhegte und entschärfte. Damit stehen wir allerdings wieder vor der eingangs gestellten Frage - oder auch: vor einer neuen Variante: Wie aber konnte es dann zu der ,Kri-

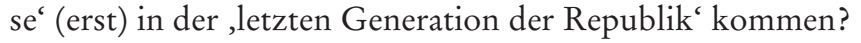

${ }^{69}$ Der Begriff stammt von Jochen Bleicken, Das Volkstribunat der klassischen Republik. Studien zu seiner Entwicklung zwischen 287 und 133 v. Chr. (München $\left.{ }^{2} 1968\right)$ 1; $152 \mathrm{f}$.

70 Begriffe nach Hölkeskamp, Conquest, Competition and Consensus (wie Anm.24). Das ist ein wichtiges allgemeines Ergebnis der grundlegenden Arbeit von Hans Beck, Karriere und Hierarchie. Die römische Aristokratie und die Anfänge des cursus honorum in der mittleren Republik (Berlin 2005); vgl. auch seinen Beitrag in diesem Band. S. dazu neuerdings auch Bruno Bleckmann, Die römische Nobilität im Ersten Punischen Krieg. Untersuchungen zur aristokratischen Konkurrenz in der Republik (Berlin 2002). Vgl. zum (prekären) Verhältnis von ,Konkurrenz‘ und ,Konsens' bereits Hölkeskamp, Nobilität (wie Anm.22) 241ff. u.ö.; ders., Konsens und Konkurrenz (wie Anm. 62). 
